University of Windsor

Scholarship at UWindsor

\title{
8-2014
}

\section{Consistency test and weight generation for additive interval fuzzy preference relations}

\author{
Yejun Xu \\ Hohai University \\ Kevin Li \\ University of Windsor \\ Huimin Wang \\ Hohai University
}

Follow this and additional works at: https://scholar.uwindsor.ca/odettepub

Part of the Business Commons

\section{Recommended Citation}

$\mathrm{Xu}$, Yejun; Li, Kevin; and Wang, Huimin. (2014). Consistency test and weight generation for additive interval fuzzy preference relations. Soft Computing, 18 (8), 1499-1513.

https://scholar.uwindsor.ca/odettepub/91

This Article is brought to you for free and open access by the Odette School of Business at Scholarship at UWindsor. It has been accepted for inclusion in Odette School of Business Publications by an authorized administrator of Scholarship at UWindsor. For more information, please contact scholarship@uwindsor.ca. 


\title{
Consistency test and weight generation for additive interval fuzzy preference relations
}

\author{
Yejun $\mathrm{Xu}{ }^{1,2^{*}}$, Kevin W. Li ${ }^{3}$, Huimin Wang ${ }^{1,2}$ \\ ${ }^{1}$ State Key Laboratory of Hydrology-Water Resources and Hydraulic Engineering, Hohai \\ University, Nanjing, 210098, PR China \\ ${ }^{2}$ Business School, Hohai University, Jiangning, Nanjing, 211100, PR China \\ ${ }^{3}$ Odette School of Business, University of Windsor, Windsor, Ontario, Canada N9B 3P4
}

\begin{abstract}
Some simple yet pragmatic methods of consistency test are developed to check whether an interval fuzzy preference relation is consistent. Based on the definition of additive consistent fuzzy preference relations proposed by Tanino [Fuzzy Sets and Systems 12 (1984) 117131], a study is carried out to examine the correspondence between the element and weight vector of a fuzzy preference relation. Then, a revised approach is proposed to obtain priority weights from a fuzzy preference relation. A revised definition is put forward for additive consistent interval fuzzy preference relations. Subsequently, linear programming models are established to generate interval priority weights for additive interval fuzzy preference relations. A practical procedure is proposed to solve group decision problems with additive interval fuzzy preference relations. Theoretic analysis and numerical examples demonstrate that the proposed methods are more accurate than those in Xu and Chen [European Journal of Operational Research, 184 (2008) 266280].
\end{abstract}

Keywords: Multiple criteria decision analysis; interval fuzzy preference relation; consistency test; weight generation; additive consistent; linear programming.

\section{Introduction}

A fuzzy preference relation is one of the most common preference relations to express a decision-maker's (DM's) judgment information. Orlovsky (1978) defined fuzzy equivalence and strict preference relations for a given binary fuzzy non-strict preference relation and introduced two types of linearity of a fuzzy relation, thereby examining the equivalence of unfuzzy non-dominated alternatives. Tanino (1984) discussed how to use fuzzy preference orderings in group decision making and defined fuzzy preference relations as pairwise comparison matrices with additive reciprocity and max-min transitivity. Kacprzyk (1986) introduced a fuzzy majority rule characterized by a fuzzy linguistic

\footnotetext{
${ }^{*}$ Corresponding author. Tel. +86-25-68514612; fax: +86-25-85427972

E-mail address: xuyejohn@163.com(Y.-J.Xu).
} 
quantifier to investigate group decision making with a collection of individual fuzzy preference relations. Chiclana et al. (2001) studied how to integrate the multiplicative preference relations in fuzzy multipurpose decision-making problems under different preference representation structures (orderings, utilities and fuzzy preference relations), and introduced a new ordered weighted averaging (OWA) aggregation operator to aggregate multiplicative preference relations. Chiclana et al. (2003) studied conditions under which reciprocity property is maintained when aggregating preference relations using an OWA operator guided by a relative linguistic quantifier. Chiclana et al. (2007) provided some induced OWA operators to aggregate fuzzy preference relations in group decision making problems. However, a DM often cannot estimate his/her preference with exact numerical values but with interval numbers due to increasing complexity and uncertainty in real-life decision problems as well as the DM's limited attention and information processing capability. In such situations, an interval fuzzy preference relation (IFPR) becomes suitable for expressing the DM's uncertain preference information.

For IFPRs, Xu (2004c) defined a compatibility degree between two IFPRs, and demonstrated the compatibility relationships among individual and collective IFPRs. Herrera et al. (2005) developed an aggregation process for combining IFPRs with other types of information such as numerical and linguistic preference relations. Jiang (2007) introduced an index to measure the similarity degree of two IFPRs, and used an error-propagation principle to determine priority vectors for the aggregated IFPRs. $\mathrm{Xu}$ and Chen (2008b) defined additive and multiplicative consistency for IFPRs, and established linear programming models for deriving priority weights from various IFPRs. Genç et al. (2010) examined consistency, missing value(s) and derivation of priority vectors from IFPRs. Liu et al. (2012a) proposed a new method to obtain priority weights from an IFPR. Wang et al. (2012) furnished a new additive consistency definition for IFPRs, and developed linear programming models to generate interval priority weights from additive consistent or inconsistent IFPRs. Wang and Li (2012) employed interval arithmetic to define additive and multiplicative consistency of IFPRs and developed goal-programming-based models to derive interval weights from IFPRs for both individual and group decision situations. 
The aforesaid literature review indicates that weight generation is a popular approach to deriving a ranking for alternatives based on fuzzy and interval fuzzy preference relations. These weight derivation methods are typically founded upon the additive and multiplicative consistency concepts proposed by Tanino (1984) for a fuzzy preference relation $R=\left(r_{i j}\right)_{n \times n}$. For instance, Chiclana et al. (2001) provided a transformation function between multiplicative reciprocal preference relations values in the interval scale $[1 / 9,9]$ and fuzzy reciprocal preference relations with values in $[0,1]$. Herrera-Viedma et al. (2004) pointed out that the additive transitivity defined by Tanino (1984) is acceptable in characterizing consistency in fuzzy preference relations. They also showed that the consistency definition in the case of multiplicative reciprocal preference relations via the transformation function is equivalent to the additive transitivity property. Along with multiplicative consistency, additive consistency arises as parallel concept to characterize judgment consistency in fuzzy preference relations. The additive consistent property has been extended to study incomplete fuzzy preference relations (Fedrizzi and Silvio, 2007; Herrera-Viedma et al., 2007a; HerreraViedma et al., 2007b; Wu and Xu, 2012; Xu et al., 2010), complete/incomplete linguistic preference relations (Wang and Chen, 2008; Alonso et al., 2009; Dong et al., 2008; Cabrerizo et al., 2010b). Although Chiclana et al. (2009b) revealed that the multiplicative transitivity property introduced by Tanino (1984) and a generic representable uninorm function are most appropriate to depict consistency for fuzzy preference relations, it is problematic for a uninorm function to handle the cases of $(0,1)$ and $(1,0)$. As a matter of fact, the uninorm function (3) in Chiclana et al. (2009b) seems to yield counterintuitive consistent preference values when $(x, y) \rightarrow\{(0,1),(1,0)\}$. On the other hand, the additive transitivity property given by Tanino (1984) is convenient to address these cases. In this paper, we further present that the additive consistent fuzzy preference relation possesses some desired properties as given in Propositions 1 and 2. For an additive consistent fuzzy preference relation $R=\left(r_{i j}\right)_{n \times n}$, Tanino (1984) first established the correspondence between its elements and a weight vector $W=\left(w_{1}, w_{2}, \ldots, w_{n}\right)^{T}$ such that $r_{i j}=0.5+\beta\left(w_{i}-w_{j}\right) \quad($ where $\beta=0.5, i, j=1,2, \ldots$, $n$ ), but it was later shown that the correspondence is not always valid from different perspectives (Liu et al., 2012b; Xu et al., 2009; Xu et al., 2010; Fedrizzi 
and Brunelli, 2009; Fedrizzi and Brunelli, 2010; Lee and Tseng, 2006; Shen et al., 2009). $\mathrm{Xu}$ and Chen (2008b) extended the correspondence to additive consistent IFPRs. In this paper, we shall show that the parameter $\beta$ should not always be set at 0.5 , and develop revised models for deriving priority weights based on additive consistent or inconsistent IFPRs.

It is well known that a consistency test plays an important role in decision modeling with preference relations (Wang et al., 2005a). High inconsistency is likely to result in unreliable priority weights and final ranking for alternatives. Therefore, in order to ensure rationality of decisions, a preference relation should pass a consistency test before it is utilized in a decision process. However, limited work has been conducted to check whether an IFPR is consistent. This paper focuses on consistency test and weight generation from IFPRs based on the additive consistency property.

The rest of this paper is organized as follows. Section 2 provides preliminaries on fuzzy preference relations as well as some new properties of an additive consistent fuzzy preference relation. Section 3 develops some simple yet pragmatic approaches to checking whether an IFPR is additive consistent. Revised models are then presented for deriving priority weights from an additive consistent or inconsistent IFPRs. A practical procedure is also proposed to solve group decision problems with IFPRs. Section 4 furnishes a numerical example and conducts a comparative analysis between the proposed approaches and those put forth by $\mathrm{Xu}$ and Chen (2008b). Conclusions and future opportunities are discussed in Section 5.

\section{Preliminaries}

\subsection{Basic concepts of a consistent fuzzy preference relation}

For a multiple criteria decision making problem with a finite set of $n$ alternatives, let $X=\left\{x_{1}, x_{2}, \ldots, x_{n}\right\}(n \geq 2)$ be the finite set of alternatives, where $x_{i}$ denotes the $i$ th alternative. The DM often compares each pair of alternatives in $X$ and provides his/her preference degree $r_{i j}$ of alternative $x_{i}$ over $x_{j}$.

Let $W=\left(w_{1}, w_{2}, \ldots, w_{n}\right)^{T}$ be a weight vector of the alternatives, where $w_{i}$ $(i=1,2, \ldots, n)$ are nonnegative and normalized, i.e., 


$$
w_{i} \geq 0, \quad i=1,2, \ldots, n, \sum_{i=1}^{n} w_{i}=1
$$

Definition 1. Let $R=\left(r_{i j}\right)_{n \times n}$ be a preference relation, then $R$ is called a fuzzy preference relation (Kacprzyk, 1986; Chiclana et al., 1998; Chiclana et al., 2002; Fan et al., 2006; Herrera-Viedma et al., 2004; Xu et al., 2013b), if

$$
r_{i j} \in[0,1], r_{i j}+r_{j i}=1, \quad r_{i i}=0.5, \text { for all } i, j=1,2, \ldots, n
$$

Definition 2. Let $R=\left(r_{i j}\right)_{n \times n}$ be a fuzzy preference relation, then $R$ is called an additive transitive fuzzy preference relation if the following additive transitivity (Tanino, 1984) is satisfied:

$$
r_{i j}=r_{i k}+r_{k j}-0.5, \text { for all } i, j, k=1,2, \ldots, n
$$

$R$ is called a multiplicative consistent fuzzy preference relation if it satisfies the multiplicative transitivity property (Tanino, 1984):

$$
\frac{r_{j i}}{r_{i j}} \frac{r_{k j}}{r_{j k}}=\frac{r_{k i}}{r_{i k}}, \text { for all } i, j, k=1,2, \ldots, n
$$

where $r_{i j}>0, i, j=1,2, \ldots, n$.

If there exists $r_{i j}=0$, Eq. (4) can be rewritten in a multiplication form as $r_{i k} r_{k j} r_{j i}=r_{i j} r_{j k} r_{k i}$.

An additive transitive fuzzy preference relation is also referred to as additive consistent (Herrera-Viedma et al., 2004). Furthermore, additive consistency has the following desired properties:

Proposition 1. Let $R=\left(r_{i j}\right)_{n \times n}$ be an additive consistent fuzzy preference relation, then

(a) if $\lambda \geq 0.5, r_{i k} \geq \lambda, r_{k j} \geq \lambda$, then $r_{i j} \geq \lambda$;

(b) if $\lambda \leq 0.5, r_{i j} \leq \lambda, r_{j k} \leq \lambda$, then $r_{i k} \leq \lambda$.

Proof. (a) As $R=\left(r_{i j}\right)_{n \times n}$ is an additive consistent fuzzy preference relation, and $\lambda \geq 0.5$, then

$$
\begin{aligned}
r_{i j} & =r_{i k}+r_{k j}-0.5 \\
& \geq \lambda+\lambda-0.5 \\
& \geq \lambda
\end{aligned}
$$


(b) if $\lambda \leq 0.5$,

$$
\begin{aligned}
r_{i j} & =r_{i k}+r_{k j}-0.5 \\
& \leq \lambda+\lambda-0.5 \\
& \leq \lambda
\end{aligned}
$$

This property is called center-division transitivity (Yao and Zhang, 1997) and is consistent with human beings psychological cognitive characteristics. It implies that the cardinality of preference is retained by this transitivity. More specifically,

(a) if $\lambda \geq 0.5$, and $x_{i}$ is preferred to $x_{k}\left(r_{i k} \geq \lambda\right), x_{k}$ is preferred to $x_{j}$ $\left(r_{k j} \geq \lambda\right)$, then $x_{i}$ should be preferred to $x_{j}\left(r_{i j} \geq \lambda\right)$.

(b) if $\lambda \leq 0.5$, and $x_{k}$ is preferred to $x_{i}\left(r_{i k} \leq \lambda\right), x_{j}$ is preferred to $x_{k}$ $\left(r_{k j} \leq \lambda\right)$, then $x_{j}$ should be preferred to $x_{i}\left(r_{i j} \leq \lambda\right)$.

Proposition 2 (Yao and Zhang, 1997). Let $R=\left(r_{i j}\right)_{n \times n}$ be an additive consistent fuzzy preference relation, if the $i$ th row and $i$ th column are removed from $R$, then the fuzzy preference relation composed by the remainder $(n-1)$ rows and $(n-$ 1) columns of $R$ remains additive consistent.

Lemma 1 (Xu et al., 2009; Liu et al., 2012b). Let $R=\left(r_{i j}\right)_{n \times n}$ be a fuzzy preference relation, then the sum of all the elements of $R$ is $n^{2} / 2$, that is

$$
\sum_{i=1}^{n} \sum_{j=1}^{n} r_{i j}=n^{2} / 2
$$

\subsection{New properties of additive consistent fuzzy preference relations}

In the following, new properties are revealed for additive consistent fuzzy preference relations.

Theorem 1. $R$ is an additive consistent fuzzy preference relation if and only if

$$
r_{i 1}=r_{i j}+r_{j 1}-0.5, \quad \forall i, j=1,2, \ldots, n
$$

Proof. If $R$ is an additive consistent fuzzy preference relation, then

$$
r_{i k}=r_{i j}+r_{j k}-0.5, i, j, k=1,2, \ldots, n
$$

Especially, when $k=1$, Eq.(6) becomes Eq.(5).

Conversely, if Eq.(5) holds, then

$$
r_{k 1}=r_{k j}+r_{j 1}-0.5
$$




$$
\begin{aligned}
r_{i 1} & =r_{i k}+r_{k 1}-0.5 \\
& =r_{i k}+\left(r_{k j}+r_{j 1}-0.5\right)-0.5
\end{aligned}
$$

By Eqs. (5) and (8), we have

$$
r_{i j}+r_{j 1}-0.5=r_{i k}+\left(r_{k j}+r_{j 1}-0.5\right)-0.5
$$

That is

$$
r_{i j}=r_{i k}+r_{k j}-0.5
$$

By Definition 2, $R$ is an additive consistent fuzzy preference relation.

Similarly, we can get the following result.

Theorem 2. $R$ is an additive consistent fuzzy preference relation if and only if

$$
r_{1 j}=r_{1 i}+r_{i j}-0.5, \quad \forall i, j=1,2, \ldots, n
$$

Remark 1. Theorems 1 and 2 are only special cases of Definition 2. However, it is more simpler to check whether a reciprocal fuzzy preference relation is additive consistent or not. It could relief the computation burden. This property is extended to Theorems 4 and 5. From Tables 1 and 2 in Example 1, we can see that it can reduce the computational work.

Lemma 2 (Xu, 2000; Xu et al., 2009). For a complete fuzzy preference relation $R=\left(r_{i j}\right)_{n \times n}$, there exists a weight vector $W=\left(w_{1}, w_{2}, \ldots, w_{n}\right)^{T}$, such that

$$
r_{i j}=\frac{1}{2}\left(w_{i}-w_{j}+1\right)
$$

if and only if $R$ is additive consistent. Furthermore, $W=\left(w_{1}, w_{2}, \ldots, w_{n}\right)^{T}$ can be determined by

$$
w_{i}=\frac{2}{n} \sum_{k=1}^{n} r_{i k}+\frac{1}{n}-1 .
$$

Remark 2. In (Liu et al., 2012b), it was pointed out that the weight vector determined by (11) may not always satisfy Eq.(1). For instance,

$$
R=\left[\begin{array}{ccc}
0.5 & 0 & 0 \\
1 & 0.5 & 0.5 \\
1 & 0.5 & 0.5
\end{array}\right]
$$

By Definition 2, it is evident that $R$ is a consistent fuzzy preference relation. By Lemma 2, we have $w_{1}=-1 / 3, w_{2}=2 / 3, w_{3}=2 / 3$. It is shown that a negative 
weight $w_{1}<0$ is derived. It has been revealed that Eqs.(1) and (10) are incompatible from different points of view (See Fedrizzi and Brunelli (2009; 2010), Xu et al. (2009; 2010) and Lee and Tseng (2006)). In order to use Eq.(10) to reflect the relationship between the element $r_{i j}$ and the weight vector $w_{i}$, Fedrizzi and Brunelli (2009) suggested that the weight vector should not be normalized. A different angle is adopted to examine this problem: the normalization condition (1) is maintained, but the relationship between the element $r_{i j}$ and the weight vector $w_{i}$ will be revised.

Theorem 3. For a complete fuzzy preference relation $R=\left(r_{i j}\right)_{n \times n}$, if $R$ is additive consistent, there always exists a priority weight vector $W=\left(w_{1}, w_{2}\right.$, $\left.\ldots, w_{n}\right)^{T}$ such that

$$
w_{i} \geq 0 \quad(i=1,2, \ldots, n), \sum_{i=1}^{n} w_{i}=1
$$

The relationship between $w_{i}$ and $r_{i j}$ is $(\mathrm{Xu}, 2000$; $\mathrm{Xu}$ et al., 2009; Liu et al., 2012b)

$$
w_{i}=\frac{1}{2 n \beta}\left(2 \sum_{k=1}^{n} r_{i k}-n\right)+\frac{1}{n}, r_{i j}=0.5+\beta\left(w_{i}-w_{j}\right)
$$

where $\beta \geq \max \left\{\frac{n}{2}-\sum_{k=1}^{n} r_{i k}\right\}$. Furthermore, if $\sum_{k=1}^{n} r_{i k} \geq \sum_{k=1}^{n} r_{j k}$, then $w_{i} \geq w_{j}$.

Especially, (i) if $\beta=\frac{n-1}{2}, w_{i}=\frac{2 \sum_{k=1}^{n} r_{i k}-1}{n(n-1)}$; (ii) if $\beta=\frac{n}{2}, w_{i}=\frac{2 \sum_{k=1}^{n} r_{i k}}{n^{2}}$.

Proof. For an additive consistent fuzzy preference relation, by Lemma 1, we have

$$
\sum_{i=1}^{n} \sum_{k=1}^{n} r_{i k}=\frac{n^{2}}{2} \geq n \times \min \left\{\sum_{k=1}^{n} r_{i k}\right\}
$$

i.e.,

$$
\frac{n}{2} \geq \min \left\{\sum_{k=1}^{n} r_{i k}\right\}
$$

thus,

$$
\max \left\{\frac{n}{2}-\sum_{k=1}^{n} r_{i k}\right\} \geq 0
$$

Then, for the parameter $\beta$, we have 


$$
\beta \geq \max \left\{\frac{n}{2}-\sum_{k=1}^{n} r_{i k}\right\} \geq 0
$$

And it can be verified that

$$
\begin{aligned}
w_{i} & =\frac{1}{2 n \beta}\left(2 \sum_{k=1}^{n} r_{i k}-n\right)+\frac{1}{n} \\
& =\frac{\left(2 \sum_{k=1}^{n} r_{i k}-n\right)+2 \beta}{2 n \beta} \\
& =\frac{2\left[\beta-\left(\frac{n}{2}-\sum_{k=1}^{n} r_{i k}\right)\right]}{2 n \beta} \\
& \geq \frac{2\left[\max \left\{\frac{n}{2}-\sum_{k=1}^{n} r_{i k}\right\}-\left(\frac{n}{2}-\sum_{k=1}^{n} r_{i k}\right)\right]}{2 n \beta} \\
& \geq 0
\end{aligned}
$$

And with Lemma 1,

$$
\sum_{i=1}^{n} w_{i}=\sum_{i=1}^{n} \frac{1}{2 n \beta}\left(2 \sum_{k=1}^{n} r_{i k}-n\right)+1=\left(\frac{2 \sum_{i=1}^{n} \sum_{k=1}^{n} r_{i k}-n^{2}}{2 n \beta}\right)+1=\left(\frac{2 \times \frac{n^{2}}{2}-n^{2}}{2 n \beta}\right)+1=1
$$

If $R$ is additive consistent, plugging the aforesaid $w_{i}$, we have

$$
0.5+\beta\left(w_{i}-w_{j}\right)=0.5+\frac{1}{n} \sum_{k=1}^{n}\left(r_{i k}-r_{j k}\right)=0.5+\frac{1}{n} \sum_{k=1}^{n}\left(r_{i j}-\frac{1}{2}\right)=r_{i j}
$$

Furthermore, since $\beta>0$, if $\sum_{k=1}^{n} r_{i k} \geq \sum_{k=1}^{n} r_{j k}$, by the definition of $w_{i}$ in (12) , one has $w_{i} \geq w_{j}$. This shows that the ranking order is preserved regardless of the value of $\beta$. Especially, if $\beta=(n-1) / 2, w_{i}=\frac{2 \sum_{k=1}^{n} r_{i k}-1}{n(n-1)}$; If $\beta=n / 2$, $w_{i}=\frac{2 \sum_{k=1}^{n} r_{i k}}{n^{2}}$. In both cases, $\beta \geq \max \left\{\frac{n}{2}-\sum_{k=1}^{n} r_{i k}\right\} \geq 0$, and hence $w_{i} \geq 0$.

However, if $\beta=\frac{1}{2}$ in Eq.(12), $\beta=\frac{1}{2} \geq \max \left\{\frac{n}{2}-\sum_{k=1}^{n} r_{i k}\right\}$ does not always hold, leading to $w_{i}<0$ in some cases. Xu et al. (2009) set $\beta=n / 2$, and referred it as a normalizing rank aggregation method. Xu et al. (2010) assumed 
$\beta=(n-1) / 2$, it was shown that both cases yield better performance than the case when $\beta$ takes 0.5 . Theorem 3 reveals a relationship between the element $r_{i j}$ and the weight vector $w_{i}$, where the nonnegative weights are normalized to 1 . In the example given in Remark 1, if Fedrizzi and Brunelli's (2009) method is employed to compute the priority vector, one has $w_{1}=0, w_{2}=1, w_{3}=1$, resulting in a non-normalized weight vector. By Theorem 3, we have $w_{1}=\frac{1}{3}-\frac{1}{3 \beta}$, $w_{2}=w_{3}=\frac{1}{3}+\frac{1}{6 \beta}$, where $\beta \geq \max \left\{\frac{n}{2}-\sum_{k=1}^{n} r_{i k}\right\} \geq 1$. By setting different $\beta$ values in (12), one can obtain distinct normalized weight vectors satisfying Eq.(1). On the contrary, Fedrizzi and Brunelli's (2009) method can only get one weight vector. It is also shown that, by introducing the parameter $\beta$, the proposed formula (12) makes the normalization constraint Eq.(1) compatible with additive consistency.

Remark 3. In Theorem 3, $w_{i}$ can be seen as the priority weight for alternative $x_{i}$, while $\beta$ can be regarded as an adjusting parameter to obtain a normalized priority weight vector. As long as $\beta \geq \max \left\{\frac{n}{2}-\sum_{k=1}^{n} r_{i k}\right\} \geq 0$, despite that the priority weight $w_{i}$ changes with $\beta$ in Eq.(12), the ranking implied in the weight vector $W$ remains the same. On the other hand, the larger the value of $\beta$, the smaller the differences among $w_{i}$ 's. Especially, if $\beta \rightarrow+\infty, w_{i} \rightarrow \frac{1}{n}$, and there will be no difference in weights among the alternatives. To maximize the discrimination power among weights for the alternatives, one can assign $\beta$ to its lower bound as $\beta=\max \left\{\frac{n}{2}-\sum_{k=1}^{n} r_{i k}\right\}$. Liu et al. (2012b) proposed Eq.(12), the difference between this paper and Liu et al.'s is that $\beta$ herein is not a given value, while $\beta$ in Liu et al's is predetermined. Their condition can only deduce one weight vector $W$, whereas we can obtain diverse weight vectors by setting different values for $\beta$.

\section{Approaches to consistency test and weight generation for interval fuzzy preference relations}




\subsection{Consistency test for interval fuzzy preference relations}

With increasing complexity and uncertainty in real-life decision situations, DMs can hardly estimate their preferences with numerical values. Instead it is often easier and more practical for DMs to furnish their evaluations in a range of values or interval numbers. Hereafter, it is assumed that a DM compares each pair of criteria or alternatives in $X$, and provides his/her interval fuzzy preference degree $\tilde{r}_{i j}=\left[l_{i j}, u_{i j}\right]$ of $x_{i}$ over $x_{j}$, where $\tilde{r}_{i j}$ indicates that $x_{i}$ is preferred to $x_{j}$ with a preference degree between $l_{i j}$ and $u_{i j}$. All these interval fuzzy preference degrees $\tilde{r}_{i j}(i, j=1,2, \ldots, n)$ consist of IFPR $\tilde{R}=\left(\tilde{r}_{i j}\right)_{n \times n} \quad(\mathrm{Xu}, 2004 \mathrm{c})$

$$
\tilde{R}=\left(\tilde{r}_{i j}\right)_{n \times n}=\left[\begin{array}{cccc}
{\left[l_{11}, u_{11}\right]} & {\left[l_{12}, u_{12}\right]} & \ldots & {\left[l_{1 n}, u_{1 n}\right]} \\
{\left[l_{21}, u_{21}\right]} & {\left[l_{22}, u_{22}\right]} & \ldots & {\left[l_{2 n}, u_{2 n}\right]} \\
\vdots & \vdots & \vdots & \vdots \\
{\left[l_{n 1}, u_{n 1}\right]} & {\left[l_{n 2}, u_{n 2}\right]} & \ldots & {\left[l_{n n}, u_{n n}\right]}
\end{array}\right]
$$

Where $\tilde{r}_{i j}=\left[l_{i j}, u_{i j}\right], \quad \tilde{r}_{j i}=\left[l_{j i}, u_{j i}\right], l_{i j}+u_{j i}=u_{i j}+l_{j i}=1, u_{i j} \geq l_{i j} \geq 0, l_{i i}=u_{i i}=0.5$, for all $i, j=1,2, \ldots, n$.

Definition 3 (Lan et al., 2012). Let $\tilde{R}=\left(\tilde{r}_{i j}\right)_{n \times n}$ be an IFPR. If there exists an additive consistent fuzzy preference relation $R=\left(r_{i j}\right)_{n \times n}$, such that

$$
l_{i j} \leq r_{i j} \leq u_{i j}, \quad \forall i, j=1,2, \ldots, n
$$

Then $\tilde{R}$ is called an additive consistent IFPR and $R=\left(r_{i j}\right)_{n \times n}$ is called additive consistent information in $\tilde{R}$.

Theorem 4. $\tilde{R}=\left(\tilde{r}_{i j}\right)_{n \times n}$ is an additive consistent IFPR if and only if

$$
\bigcap_{j=1}^{n}\left(\left[l_{i j}, u_{i j}\right]-\left[l_{1 j}, u_{1 j}\right]\right) \neq \varnothing, \quad i=1,2, \ldots, n
$$

i.e., it satisfies the following inequality constraints:

$$
\max _{j}\left(l_{i j}+l_{j 1}-0.5\right) \leq \min _{j}\left(u_{i j}+u_{j 1}-0.5\right), \quad \forall i=1,2, \ldots, n
$$

Proof. Necessity: Assume $\tilde{R}=\left(\tilde{r}_{i j}\right)_{n \times n}$ is an additive consistent IFPR, there exists an additive consistent fuzzy preference relation $R=\left(r_{i j}\right)_{n \times n}$, such that

$$
l_{i j} \leq r_{i j} \leq u_{i j}, \quad \forall i, j=1,2, \ldots, n,
$$

Especially, when $i=1$,

$$
l_{1 j} \leq r_{1 j} \leq u_{1 j}, \quad \forall j=1,2, \ldots, n
$$


For any $i=1,2, \ldots, n$,

$$
l_{i j}-u_{1 j} \leq r_{i j}-r_{1 j} \leq u_{i j}-l_{1 j}, \quad \forall j=1,2, \ldots, n
$$

Since $R=\left(r_{i j}\right)_{n \times n}$ is an additive consistent fuzzy preference relation, then

$$
r_{i j}=r_{1 j}-r_{1 i}+0.5, \quad \forall i, j=1,2, \ldots, n
$$

Eq.(18) can be changed into

$$
l_{i j}-u_{1 j} \leq-r_{1 i}+0.5 \leq u_{i j}-l_{1 j}, \quad \forall j=1,2, \ldots, n
$$

Therefore,

$$
-r_{1 i}+0.5 \in \bigcap_{j=1}^{n}\left[l_{i j}-u_{1 j}, u_{i j}-l_{1 j}\right]=\bigcap_{j=1}^{n}\left(\left[l_{i j}, u_{i j}\right]-\left[l_{1 j}, u_{1 j}\right]\right)
$$

Thus, $\bigcap_{j=1}^{n}\left(\left[l_{i j}, u_{i j}\right]-\left[l_{1 j}, u_{1 j}\right]\right) \neq \varnothing$.

Since $u_{1 j}=1-l_{j 1}, l_{1 j}=1-u_{j 1}$, then $\bigcap_{j=1}^{n}\left[l_{i j}+l_{j 1}-1, u_{i j}+u_{j 1}-1\right] \neq \varnothing$,

therefore,

$$
\bigcap_{j=1}^{n}\left[l_{i j}+l_{j 1}-0.5, u_{i j}+u_{j 1}-0.5\right] \neq \varnothing
$$

i.e., $\max _{j}\left(l_{i j}+l_{j 1}-0.5\right) \leq \min _{j}\left(u_{i j}+u_{j 1}-0.5\right), \quad \forall i=1,2, \ldots, n$.

Sufficiency: if $\bigcap_{j=1}^{n}\left(\left[l_{i j}, u_{i j}\right]-\left[l_{1 j}, u_{1 j}\right]\right) \neq \varnothing$, i.e., Eq.(15) holds,

$u_{1 j}=1-l_{j 1}, l_{1 j}=1-u_{j 1}$, then $\bigcap_{j=1}^{n}\left[l_{i j}+l_{j 1}-1, u_{i j}+u_{j 1}-1\right] \neq \varnothing$,

assume that

$$
\bigcap_{j=1}^{n}\left[l_{i j}+l_{j 1}-1, u_{i j}+u_{j 1}-1\right]=\left[p_{i 1}^{-}, p_{i 1}^{+}\right]
$$

then

$$
p_{i 1}^{-}=\max _{j}\left(l_{i j}+l_{j 1}-1\right), \quad p_{i 1}^{+}=\min _{j}\left(u_{i j}+u_{j 1}-1\right), \quad \forall i=2, \ldots, n
$$

let

$$
\begin{aligned}
& p_{1 j}^{-}=\max _{i}\left\{l_{1 j}, l_{i j}-p_{i 1}^{+}\right\}, \quad p_{1 j}^{+}=\min _{i}\left\{u_{1 j}, u_{i j}-p_{i 1}^{-}\right\} \\
& p_{i j}^{-}=p_{1 j}^{+}+p_{i 1}^{-}, \quad p_{i j}^{+}=p_{1 j}^{-}+p_{i 1}^{+}, \quad \forall i=2, \ldots, n \\
& p_{1 j}=\frac{p_{1 j}^{+}+p_{1 j}^{-}}{2}, \quad p_{i 1}=\frac{p_{i 1}^{-}+p_{i 1}^{+}}{2}+0.5, \\
& p_{i j}=\frac{p_{i j}^{-}+p_{i j}^{+}}{2}
\end{aligned}
$$


then

$$
\begin{aligned}
p_{i j} & =\frac{p_{1 j}^{+}+p_{i 1}^{-}+p_{1 j}^{-}+p_{i 1}^{+}}{2}=\frac{\left(p_{1 j}^{-}+p_{1 j}^{+}\right)+\left(p_{i 1}^{-}+p_{i 1}^{+}\right)}{2} \\
& =p_{1 j}+p_{i 1}-0.5
\end{aligned}
$$

i.e., there exist a fuzzy preference relation $P$ such that

$$
p_{i 1}=p_{i j}+p_{j 1}-0.5, \forall i, j=1,2, \ldots, n
$$

By Theorem 1, $P$ is an additive consistent fuzzy preference relation, and by Definition 3, $\tilde{R}=\left(\tilde{r}_{i j}\right)_{n \times n}$ is an additive consistent IFPR. The proof of Theorem 4 is thus completed.

Example 1 is presented in Section 4 to show how an additive fuzzy preference relation is constructed by using aforesaid process.

Remark 4. When $i=1, \bigcap_{j=1}^{n}\left(\left[l_{1 j}, u_{1 j}\right]-\left[l_{1 j}, u_{1 j}\right]\right) \neq \varnothing$ (i.e., Eq.(14)) always holds. Similarly, we have:

Theorem 5. If $\tilde{R}=\left(\tilde{r}_{i j}\right)_{n \times n}$ is an additive consistent IFPR, then for any

$$
\begin{gathered}
j=1,2, \ldots, n, \bigcap_{i=1}^{n}\left(\left[l_{i j}, u_{i j}\right]-\left[l_{i 1}, u_{i 1}\right]\right) \neq \varnothing, \text { i.e. }, \\
\max _{i}\left(l_{i j}+l_{1 i}-0.5\right) \leq \min _{i}\left(u_{i j}+u_{1 i}-0.5\right), \quad \forall j=1,2, \ldots, n
\end{gathered}
$$

Theorem 6. $\tilde{R}=\left(\tilde{r}_{i j}\right)_{n \times n}$ is an additive consistent IFPR if and only if it satisfies the following inequality constraints:

$$
\max _{j}\left(l_{i j}+l_{j k}-0.5\right) \leq \min _{j}\left(u_{i j}+u_{j k}-0.5\right), \quad \forall i, j, k=1,2, \ldots, n
$$

Proof. If $\tilde{R}$ is an additive consistent IFPR, then there exists an additive consistent fuzzy preference relation $R=\left(r_{i j}\right)_{n \times n}$ such that

$$
\begin{gathered}
l_{i j} \leq r_{i j} \leq u_{i j}, \quad i, j=1,2, \ldots, n \\
l_{j k} \leq r_{j k} \leq u_{j k}, \quad j, k=1,2, \ldots, n
\end{gathered}
$$

By (29) and (30), we have

$$
l_{i j}+l_{j k}-0.5 \leq r_{i j}+r_{j k}-0.5 \leq u_{i j}+u_{j k}-0.5, \quad \forall i, j, k=1,2, \ldots, n
$$

Since (31) holds for any $j=1, \ldots, n$, it follows that $\max _{j}\left(l_{i j}+l_{j k}-0.5\right) \leq$ $\min _{j}\left(u_{i j}+u_{j k}-0.5\right)$ holds for all $\forall i, j, k=1,2, \ldots, n$. 
Conversely, if (28) holds for $\forall i, j, k=1,2, \ldots, n$, then there exists a fuzzy preference relation $R=\left(r_{i j}\right)_{n \times n}$ such that $l_{i k} \leq r_{i k} \leq u_{i k}$, for all $i, k=1,2, \ldots, n$. By Definition 3, $\tilde{R}$ is an additive consistent IFPR.

Theorem 7. $\tilde{R}=\left(\tilde{r}_{i j}\right)_{n \times n}$ is an additive consistent IFPR if and only if it satisfies

$$
\bigcap_{j=1}^{n}\left(\tilde{r}_{i j}+\tilde{r}_{j k}-0.5\right) \neq \varnothing, \quad \forall i, j, k=1,2, \ldots, n
$$

Proof. Necessity: If $\tilde{R}=\left(\tilde{r}_{i j}\right)_{n \times n}$ is an additive consistent IFPR, by Definition 3, there exists an additive consistent fuzzy preference relation $R=\left(r_{i j}\right)_{n \times n}$ such that $l_{i j} \leq r_{i j} \leq u_{i j}, \forall i, j=1,2, \ldots, n$, that is, for all $i, j, k=1,2, \ldots, n, \quad r_{i k}=r_{i j}+r_{j k}-0.5$ $\in \tilde{r}_{i j}+\tilde{r}_{j k}-0.5$. Thus, for all $i, j, k=1,2, \ldots, n, \bigcap_{j=1}^{n}\left(\tilde{r}_{i j}+\tilde{r}_{j k}-0.5\right) \neq \varnothing$.

Sufficiency: If $\bigcap_{j=1}^{n}\left(\tilde{r}_{i j}+\tilde{r}_{j k}-0.5\right) \neq \varnothing, \forall i, k=1,2, \ldots, n$, assume that

$$
\bigcap_{j=1}^{n}\left(\tilde{r}_{i j}+\tilde{r}_{j k}-0.5\right)=\left[p_{i j}^{-}, p_{i j}^{+}\right]
$$

It is obvious that there exists $\max _{j}\left(l_{i j}+l_{j k}-0.5\right) \leq p_{i j}^{-} \leq p_{i j}^{+} \leq \min _{j}\left(u_{i j}+u_{j k}-0.5\right)$.

By Theorem 6, $\tilde{R}=\left(\tilde{r}_{i j}\right)_{n \times n}$ is an additive consistent IFPR, which completes the proof of Theorem 7 .

Remark 5. Theorems 4 and 5 can be regarded as special cases of Theorems 6 and 7 where $k=1$. But Theorems 4 and 5 are simpler than Theorems 6 and 7 due to their computation convenience. Theorems 4-7 can be used to judge whether an IFPR is consistent without solving any mathematical programming model. It only requires simple algebraic operations. This feature will be illustrated in the numerical examples in Section 4. Since an IFPR is reciprocal in nature, only its upper or lower triangular elements have to be checked.

\subsection{Linear programming models for generating weights}

Definition 4. Let $\tilde{R}=\left(\tilde{r}_{i j}\right)_{n \times n}$ be an IFPR, where $\tilde{r}_{i j}=\left[l_{i j}, u_{i j}\right]$, for all $i, j=1,2$, $\ldots, n$, if there exists a vector $W=\left(w_{1}, w_{2}, \ldots, w_{n}\right)^{T}$ and $\beta>0$, such that

$$
l_{i j} \leq 0.5+\beta\left(w_{i}-w_{j}\right) \leq u_{i j}, \text { for all } i, j=1,2, \ldots, n
$$


where $W$ satisfies the condition (1), then $\tilde{R}$ is called an additive consistent IFPR.

Based on Theorem 3, it is easy to know that Definition 3 is equivalent to Definition 4.

Definition 5. Let $\tilde{R}=\left(\tilde{r}_{i j}\right)_{n \times n}$ be an interval fuzzy preference relation, where $\tilde{r}_{i j}=\left[l_{i j}, u_{i j}\right]$, for all $i, j=1,2, \ldots, n$, if there exists a vector $W=\left(w_{1}, w_{2}, \ldots, w_{n}\right)^{T}$ and $\beta>0$, such that

$$
l_{i j} \leq 0.5+\beta\left(w_{i}-w_{j}\right) \leq u_{i j}, \text { for all } i=1,2, \ldots, n-1 ; j=i+1, \ldots, n .
$$

where $W$ satisfies the condition (1), then $\tilde{R}$ is called an additive consistent IFPR.

Remark 6. Xu and Chen (2008b) defined the additive consistent IFPR by setting $\beta=1 / 2$ in Eqs. (33) and (34), while our definition does not require $\beta$ to be a specific value as long as it is positive. As one can see in the numerical examples in Section 4, when $\beta$ takes different values, different weight vectors $W$ are obtained accordingly, and then by Eq. (12), different fuzzy preference relations $R=\left(r_{i j}\right)_{n \times n}$, satisfying Eq. (13), are derived. However, if Xu and Chen's method is adopted by setting $\beta=1 / 2$, it is possible that there does not exist any weight vector satisfying Eq. (33), asserting an inconsistent fuzzy preference relation. On the other hand, if our definition is utilized, by increasing $\beta$, one could find corresponding weight vectors and fuzzy preference relations, leading to a consistent relation (see Examples 1 and 2 in Section 4 for details).

By the definition of IFPR, it is easy to prove the equivalence between Definitions 4 and 5. Definition 5 shows that it is only necessary to check the upper or lower triangular elements of an IFPR for additive consistency.

Remark 7. In Definition $4, \beta$ is a positive parameter. By Theorem 3 , if $\tilde{R}$ is an additive consistent IFPR, then there exists at least one additive consistent fuzzy preference relation $R=\left(r_{i j}\right)_{n \times n}$ satisfying Eq.(13), then there exists a weight vector $W=\left(w_{1}, w_{2}, \ldots, w_{n}\right)^{T}$ and $\beta>0$, satisfying $r_{i j}=0.5+\beta\left(w_{i}-w_{j}\right)$, this means that Eq.(33) holds. On the other hand, if $\tilde{R}$ is not an additive consistent IFPR, then there does not exist any additive consistent fuzzy preference relation 
$R=\left(r_{i j}\right)_{n \times n}$ satisfying Eq.(13). This means that there does not exist any vector $W=\left(w_{1}, w_{2}, \ldots, w_{n}\right)^{T}$ satisfying Eq.(33).

If $\tilde{R}=\left(\tilde{r}_{i j}\right)_{n \times n}$ is an additive consistent IFPR, then the priority vector $W=$ $\left(w_{1}, w_{2}, \ldots, w_{n}\right)^{T}$ generated from $\tilde{R}=\left(\tilde{r}_{i j}\right)_{n \times n}$ should satisfy the conditions Eq.(1) and Eq.(34). In general, for each $\beta$, a priority weight vector can be obtained, therefore, as long as such a priority vector satisfying (1) and (34) is not unique, each weight $w_{i}(i=1,2, \ldots, n)$ should fall within a range. As a result, based on the conditions (1) and (34), the following two linear programming models are established:

$(\mathrm{M}-1)$

$$
\begin{aligned}
& \qquad w_{i}^{-}=\min w_{i} \\
& \text { s.t. }\left\{\begin{array}{l}
\beta\left(w_{i}-w_{j}\right)+0.5 \geq l_{i j}, i=1,2, \ldots, n-1 ; j=i+1, \ldots, n, \\
\beta\left(w_{i}-w_{j}\right)+0.5 \leq u_{i j}, i=1,2, \ldots, n-1 ; j=i+1, \ldots, n, \\
\sum_{i=1}^{n} w_{i}=1, \\
w_{i} \geq 0, i=1,2, \ldots, n .
\end{array}\right.
\end{aligned}
$$

$$
\begin{gathered}
w_{i}^{+}=\max w_{i} \\
\text { s.t. }\left\{\begin{array}{l}
\beta\left(w_{i}-w_{j}\right)+0.5 \geq l_{i j}, i=1,2, \ldots, n-1 ; j=i+1, \ldots, n, \\
\beta\left(w_{i}-w_{j}\right)+0.5 \leq u_{i j}, i=1,2, \ldots, n-1 ; j=i+1, \ldots, n, \\
\sum_{i=1}^{n} w_{i}=1, \\
w_{i} \geq 0, i=1,2, \ldots, n .
\end{array}\right.
\end{gathered}
$$

Solving the models (M-1) and (M-2) yields interval priority weights $\tilde{w}_{i}=\left[w_{i}^{-}, w_{i}^{+}\right], \quad i=1,2, \ldots, n$.

Generally, $\tilde{R}=\left(\tilde{r}_{i j}\right)_{n \times n}$ provided by the DM is not always additive consistent, in other words, there may not exist any vector $W=\left(w_{1}, w_{2}, \ldots, w_{n}\right)^{T}$ and $\beta>0$ such that Eq.(34) holds, and no feasible solution exists for the models (M-1) and (M-2). In this case, similar to the idea in Wang et al. (2005b), Xu and Chen (2008b), the models (M-1) and (M-2) are extended to accommodate inconsistent judgments by introducing deviation variables $d_{i j}^{-}$and $d_{i j}^{+}$for (34), $i=1,2, \ldots, n-1 ; j=i+1, \ldots, n$ :

$$
l_{i j}-d_{i j}^{-} \leq 0.5+\beta\left(w_{i}-w_{j}\right) \leq u_{i j}+d_{i j}^{+}, \text {for } i=1,2, \ldots, n-1, \quad j=i+1, \ldots, n
$$


where $d_{i j}^{-}$and $d_{i j}^{+}$are both non-negative. Especially, if both $d_{i j}^{-}$and $d_{i j}^{+}$are equal to zero, then the condition (35) is reduced to (34).

Obviously, the smaller the deviation variables $d_{i j}^{-}$and $d_{i j}^{+}$, the closer the $\tilde{R}$ to an additive consistent IFPR. As a result, the following optimization model is put forward:

$$
\begin{array}{ll}
\text { (M-3) } & J=\min \sum_{i=1}^{n-1} \sum_{j=i+1}^{n}\left(d_{i j}^{-}+d_{i j}^{+}\right) \\
\left\{\begin{array}{l}
\beta\left(w_{i}-w_{j}\right)+0.5+d_{i j}^{-} \geq l_{i j}, i=1,2, \ldots, n-1 ; j=i+1, \ldots, n, \\
\beta\left(w_{i}-w_{j}\right)+0.5-d_{i j}^{+} \leq u_{i j}, i=1,2, \ldots, n-1 ; j=i+1, \ldots, n, \\
\sum_{i=1}^{n} w_{i}=1, \\
w_{i} \geq 0, i=1,2, \ldots, n . \\
d_{i j}^{-}, d_{i j}^{+} \geq 0, i=1,2, \ldots, n-1 ; j=i+1, \ldots, n .
\end{array}\right.
\end{array}
$$

Solving this model, one can get optimal deviation values $\dot{d}_{i j}^{-}$and $\dot{d}_{i j}^{+}$, $i=1,2, \ldots, n-1 ; j=i+1, \ldots, n$. From the model (M-3), we can get the following result easily:

Theorem 9. $\tilde{R}$ is an additive consistent IFPR if and only if there exists a weight vector $W=\left(w_{1}, w_{2}, \ldots, w_{n}\right)^{T}$ and $\beta>0$ such that $J=0$.

Remark 8. Similar to Remark 6, the difference between (M-1), (M-2), Theorem 9 and $\mathrm{Xu}$ and Chen's (2008b) model is that our model allows $\beta$ to be set at different values other than $\beta=0.5$ only in $\mathrm{Xu}$ and Chen's model. It is apparent that $\mathrm{Xu}$ and Chen (2008b) imposes a more restrictive consistency condition, while our model is more relaxed in finding a weight vector satisfying Eq. (33) by setting a different value of $\beta$ than 0.5 . Examples 1 and 2 in Section 4 verify these points.

If $\tilde{R}$ is not an additive consistent IFPR, then based on the optimal deviation values $\dot{d}_{i j}^{-}$and $\dot{d}_{i j}^{+}, i=1,2, \ldots, n-1 ; j=i+1, \ldots, n$, similar to the models (M-1) and (M-2), we further establish the following two linear programming models: (M-4) $w_{i}^{-}=\min w_{i}$ 
s.t. $\left\{\begin{array}{l}\beta\left(w_{i}-w_{j}\right)+0.5+\dot{d}_{i j}^{-} \geq l_{i j}, i=1,2, \ldots, n-1 ; j=i+1, \ldots, n, \\ \beta\left(w_{i}-w_{j}\right)+0.5-\dot{d}_{i j}^{+} \leq u_{i j}, i=1,2, \ldots, n-1 ; j=i+1, \ldots, n, \\ \sum_{i=1}^{n} w_{i}=1, \\ w_{i} \geq 0, i=1,2, \ldots, n .\end{array}\right.$

$$
\begin{aligned}
& \quad w_{i}^{+}=\max w_{i} \\
& \text { s.t. }\left\{\begin{array}{l}
\beta\left(w_{i}-w_{j}\right)+0.5+\dot{d}_{i j}^{-} \geq l_{i j}, i=1,2, \ldots, n-1 ; j=i+1, \ldots, n, \\
\beta\left(w_{i}-w_{j}\right)+0.5-\dot{d}_{i j}^{+} \leq u_{i j}, i=1,2, \ldots, n-1 ; j=i+1, \ldots, n, \\
\sum_{i=1}^{n} w_{i}=1, \\
w_{i} \geq 0, i=1,2, \ldots, n .
\end{array}\right.
\end{aligned}
$$

Solving the models (M-4) and (M-5) generates the interval priority weights $\tilde{w}_{i}=\left[w_{i}^{-}, w_{i}^{+}\right], \quad i=1,2, \ldots, n$.

From the aforesaid analysis, we know that the priority weights derived from an interval fuzzy preference relation are in the form of interval numbers. Let $\tilde{w}_{i}=\left[w_{i}^{-}, w_{i}^{+}\right]$and $\tilde{w}_{j}=\left[w_{j}^{-}, w_{j}^{+}\right]$be any two interval weights, where $0 \leq w_{i}^{-}$ $\leq w_{i}^{+} \leq 1, \quad 0 \leq w_{j}^{-} \leq w_{j}^{+} \leq 1$, then the degree of possibility of $\tilde{w}_{i} \geq \tilde{w}_{j}$ is defined as (Wang et al., 2005b):

$$
p\left(\tilde{w}_{i} \geq \tilde{w}_{j}\right)=\frac{\max \left\{0, w_{i}^{+}-w_{j}^{-}\right\}-\max \left\{0, w_{i}^{-}-w_{j}^{+}\right\}}{w_{i}^{+}-w_{i}^{-}+w_{j}^{+}-w_{j}^{-}}
$$

For other equivalent forms of possibility degrees, readers are referred to $\mathrm{Xu}$ and Chen, 2008b).

\subsection{Application to group decision making with IFPRs}

Let $X=\left\{x_{1}, x_{2}, \ldots, x_{n}\right\}$ be a finite set of alternatives, $E=\left\{e_{1}, e_{2}, \ldots, e_{m}\right\}$ be a finite set of DMs, and $\lambda=\left(\lambda_{1}, \lambda_{2}, \ldots, \lambda_{m}\right)^{T}$ be the weight vector of DMs, such that $\sum_{k=1}^{m} \lambda_{k}=1$, and $\lambda_{k} \geq 0(k=1,2, \ldots, m)$. Suppose that the $m$ DMs provide their preference relations over the $n$ decision alternatives as $\tilde{R}^{(k)}=\left(\tilde{r}_{i j}\right)_{n \times n}, \quad k=1,2$, $\ldots, m$, where $\tilde{r}_{i j}=\left[l_{i j}, u_{i j}\right], \quad \tilde{r}_{j i}=\left[l_{j i}, u_{j i}\right], l_{i j}+u_{j i}=u_{i j}+l_{j i}=1, \quad 1 \geq u_{i j} \geq l_{i j} \geq 0$, $l_{i i}=u_{i i}=0.5$, for all $i, j=1,2, \ldots, n$. 
$\mathrm{Xu}(2004 \mathrm{c})$ presented that a combination of IFPRs is also an IFPR. Next, we further obtain the following result.

Theorem 10. Let $\tilde{R}^{(k)}(k=1,2, \ldots, m)$ be $m$ additive consistent IFPRs provided by the DMs, $\lambda=\left(\lambda_{1}, \lambda_{2}, \ldots, \lambda_{m}\right)^{T}$ be the weight of DMs, where $\lambda_{k} \geq 0 \quad(k=1,2$, $\ldots, m), \sum_{k=1}^{m} \lambda_{k}=1$, then the weighted average

$$
\tilde{R}=\lambda_{1} \tilde{R}^{(1)}+\lambda_{2} \tilde{R}^{(2)}+\ldots+\lambda_{m} \tilde{R}^{(m)}
$$

is also an additive consistent IFPR.

Proof. Let

$$
\begin{aligned}
\tilde{R} & =\lambda_{1} \tilde{R}^{(1)}+\lambda_{2} \tilde{R}^{(2)}+\ldots+\lambda_{m} \tilde{R}^{(m)} . \\
& =\left(\tilde{r}_{i j}\right)_{n \times n}
\end{aligned}
$$

where $\tilde{r}_{i j}=\left[l_{i j}, u_{i j}\right]=\left[\sum_{k=1}^{m} \lambda_{k} l_{i j}^{(k)}, \sum_{k=1}^{m} \lambda_{k} u_{i j}^{(k)}\right]$.

Since $\tilde{R}^{(k)}(k=1,2, \ldots, m)$ are $m$ additive consistent IFPRs, then there exist $m$ additive consistent fuzzy preference relations $R^{(k)}=\left(r_{i j}^{(k)}\right)_{n \times n}(k=1,2, \ldots, m)$ such that

$$
l_{i j}^{(k)} \leq r_{i j}^{(k)} \leq u_{i j}^{(k)}, \quad k=1,2, \ldots, m, \quad \forall i, j=1,2, \ldots, n
$$

Therefore,

$$
\sum_{k=1}^{m} \lambda_{k} l_{i j}^{(k)} \leq \sum_{k=1}^{m} \lambda_{k} r_{i j}^{(k)} \leq \sum_{k=1}^{m} \lambda_{k} u_{i j}^{(k)}
$$

Let $R=\left(r_{i j}\right)_{n \times n}$, where $r_{i j}=\sum_{k=1}^{m} \lambda_{k} r_{i j}^{(k)}$.

Then Eq.(40) can be rewritten as:

$$
l_{i j} \leq r_{i j} \leq u_{i j}
$$

By Definition 3, $\tilde{R}$ is an additive consistent IFPR. This completes the proof.

In the following, an algorithm is presented to solve group decision problems with IFPRs:

Step 1: For a group decision problem, each DM provides his/her IFPR $\tilde{R}^{(k)}=$ $\left(\tilde{r}_{i j}^{(k)}\right)_{n \times n}$, where $\tilde{r}_{i j}=\left[l_{i j}, u_{i j}\right], \quad \tilde{r}_{j i}=\left[l_{j i}, u_{j i}\right], l_{i j}+u_{j i}=u_{i j}+l_{j i}=1, u_{i j} \geq l_{i j} \geq 0, l_{i i}=$ $u_{i i}=0.5$, for all $i, j=1,2, \ldots, n$. 
Step 2. Utilize the commonly used additive weighted averaging operator (AWA) (Eq.(37)) to aggregate all opinions into a collective interval fuzzy preference relation $\tilde{R}$.

Step 3. Utilize model (M-3) to derive optimal deviation values. Then, utilize models M-4 and M-5 to derive the interval priority weights $\tilde{w}_{i}(i=1,2, \ldots, n)$ (Generally setting $\beta=(n-1) / 2$ or $\beta=n / 2)$.

Step 4. Apply Eq.(36) to compare each $\tilde{w}_{i}$ with $\tilde{w}_{j}(j=1,2, \ldots, n)$. Denote $p_{i j}=p\left(\tilde{w}_{i} \geq \tilde{w}_{j}\right)$, a complementary matrix can be derived as $P=\left(p_{i j}\right)_{n \times n}$, where

$$
p_{i j} \geq 0, \quad p_{i j}+p_{j i}=1, \quad p_{i i}=0.5, \quad i, j=1,2, \ldots, n
$$

Utilize the normalizing rank aggregation method (Xu et al., 2009)

$$
v_{i}=\frac{\sum_{j=1}^{n} p_{i j}}{n^{2} / 2}, \quad i=1,2, \ldots, n
$$

to derive a priority vector $\quad v=\left(v_{1}, v_{2}, \ldots, v_{n}\right)^{T}$ of the complementary matrix $P$.

Then, $\tilde{w}_{i}(i=1,2, \ldots, n)$ can be ranked in a descending order as per the values of $v_{i}(i=1,2, \ldots, n)$.

Step 5. End.

\section{Numerical examples and comparative analysis}

Example 1. As a premier scenic attraction in China, Jiuzhaigou Valley has been attracting an increasing number of tourists every year (adapted from (Wang et al., 2012)). One of the challenges faced by the tourism authority is to plan the traffic so that a balanced distribution of tourists can be achieved among different scenic areas. Four alternatives were proposed to address this issue, denoted as $X=\left\{x_{1}, x_{2}, x_{3}, x_{4}\right\}$. Jiuzhaigou Valley authority compared these four programs and constructed the following IFPR $\tilde{R}$ (Wang et al., 2012):

$$
\tilde{R}=\left[\begin{array}{llll}
{[0.50,0.50]} & {[0.39,0.41]} & {[0.29,0.31]} & {[0.19,0.21]} \\
{[0.59,0.61]} & {[0.50,0.50]} & {[0.39,0.41]} & {[0.29,0.31]} \\
{[0.69,0.71]} & {[0.59,0.61]} & {[0.50,0.50]} & {[0.39,0.41]} \\
{[0.79,0.81]} & {[0.69,0.71]} & {[0.59,0.61]} & {[0.50,0.50]}
\end{array}\right]
$$


Table 1. Consistency test for Example 1 by Eq.(28) of Theorem 6

\begin{tabular}{lllllll}
\hline $\begin{array}{l}\text { Judgment } \\
\text { element }\end{array}$ & $i$ & $k$ & $j$ & $l_{i j}+l_{j k}-0.5$ & $u_{i j}+u_{j k}-0.5$ & Consistency test \\
\hline & 1 & 2 & 1 & 0.39 & 0.41 & $\max \left(l_{i j}+l_{j k}-0.5\right)=0.39$ \\
$\tilde{r}_{12}$ & 1 & 2 & 3 & 0.38 & 0.42 & $\min \left(u_{i j}+u_{j k}-0.5\right)=0.41$ \\
& 1 & 2 & 4 & 0.38 & 0.42 & $\operatorname{passed}$ \\
& 1 & 3 & 1 & 0.29 & 0.31 & $\max \left(l_{i j}+l_{j k}-0.5\right)=0.29$ \\
$\tilde{r}_{13}$ & 1 & 3 & 2 & 0.28 & 0.32 & $\min \left(u_{i j}+u_{j k}-0.5\right)=0.31$ \\
& 1 & 3 & 4 & 0.28 & 0.32 & $\operatorname{passed}$ \\
& 1 & 4 & 1 & 0.19 & 0.21 & $\max \left(l_{i j}+l_{j k}-0.5\right)=0.19$ \\
$\tilde{r}_{14}$ & 1 & 4 & 2 & 0.18 & 0.22 & $\min \left(u_{i j}+u_{j k}-0.5\right)=0.21$ \\
& 1 & 4 & 3 & 0.18 & 0.22 & $\operatorname{passed}$ \\
& 2 & 3 & 1 & 0.38 & 0.42 & $\max \left(l_{i j}+l_{j k}-0.5\right)=0.39$ \\
$\tilde{r}_{23}$ & 2 & 3 & 2 & 0.39 & 0.41 & $\min \left(u_{i j}+u_{j k}-0.5\right)=0.41$ \\
& 2 & 3 & 4 & 0.38 & 0.42 & $\operatorname{passed}$ \\
& 2 & 4 & 1 & 0.28 & 0.32 & $\max \left(l_{i j}+l_{j k}-0.5\right)=0.29$ \\
$\tilde{r}_{24}$ & 2 & 4 & 2 & 0.29 & 0.31 & $\min \left(u_{i j}+u_{j k}-0.5\right)=0.31$ \\
& 2 & 4 & 3 & 0.28 & 0.32 & $\operatorname{passed}$ \\
& 3 & 4 & 1 & 0.38 & 0.42 & $\max \left(l_{i j}+l_{j k}-0.5\right)=0.39$ \\
$\tilde{r}_{34}$ & 3 & 4 & 2 & 0.38 & 0.42 & $\min \left(u_{i j}+u_{j k}-0.5\right)=0.41$ \\
& 3 & 4 & 3 & 0.39 & 0.41 & $\operatorname{passed}$ \\
\hline & & & & & &
\end{tabular}

Table 2. Consistency test for Example 1 by Eq.(15) of Theorem 4

\begin{tabular}{llllll}
\hline Judgment element & $i$ & $j$ & $l_{i j}+l_{j 1}-0.5$ & $u_{i j}+u_{j 1}-0.5$ & Consistency test \\
\hline \multirow{2}{*}{$\tilde{r}_{21}$} & 2 & 1 & 0.59 & 0.61 & $\max \left(l_{i j}+l_{j 1}-0.5\right)=0.59$ \\
& 2 & 3 & 0.58 & 0.62 & $\min \left(u_{i j}+u_{j 1}-0.5\right)=0.61$ \\
& 2 & 4 & 0.58 & 0.62 & $\operatorname{passed}$ \\
& & & & & \\
$\tilde{r}_{31}$ & 3 & 1 & 0.69 & 0.71 & $\max \left(l_{i j}+l_{j 1}-0.5\right)=0.69$ \\
& 3 & 2 & 0.68 & 0.72 & $\min \left(u_{i j}+u_{j 1}-0.5\right)=0.71$ \\
& 3 & 4 & 0.68 & 0.72 & $\operatorname{passed}$ \\
$\tilde{r}_{41}$ & 4 & 1 & 0.79 & 0.81 & $\max \left(l_{i j}+l_{j 1}-0.5\right)=0.79$ \\
& 4 & 2 & 0.78 & 0.82 & $\min \left(u_{i j}+u_{j 1}-0.5\right)=0.81$ \\
\hline
\end{tabular}


Table 3. Interval weights

\begin{tabular}{ccccc}
\hline$\beta$ & $\tilde{w}_{1}$ & $\tilde{w}_{2}$ & $\tilde{w}_{3}$ & $\tilde{w}_{4}$ \\
\hline 0.57 & {$[0,0]$} & {$[0.1579,0.1579]$} & {$[0.3333,0.3333]$} & {$[0.5088,0.5088]$} \\
1.5 & {$[0.145,0.155]$} & {$[0.2117,0.2217]$} & {$[0.2783,0.2833]$} & {$[0.345,0.355]$} \\
2 & {$[0.1713,0.1788]$} & {$[0.2213,0.2288]$} & {$[0.2713,0.2788]$} & {$[0.3213,0.3288]$} \\
10 & {$[0.2343,0.2358]$} & {$[0.2443,0.2457]$} & {$[0.2543,0.2558]$} & {$[0.2643,0.2658]$} \\
100 & {$[0.2484,0.2485]$} & {$[0.2494,0.2495]$} & {$[0.2504,0.2506]$} & {$[0.2514,0.2516]$} \\
1000 & {$[0.2498,0.2499]$} & {$[0.2499,0.2500]$} & {$[0.2500,0.2501]$} & {$[0.2501,0.2502]$} \\
10000 & {$[0.2500,0.2500]$} & {$[0.2500,0.2500]$} & {$[0.2500,0.2500]$} & {$[0.2500,0.2500]$} \\
\hline
\end{tabular}

If Eq.(14) is employed to test the consistency for $\tilde{R}$, according to Remark 3, it always holds when $i=1$, so the remaining calculation is carried out for $i=2,3$, and 4 only:

$$
\begin{aligned}
& \bigcap_{j=1}^{4}\left(\left[l_{2 j}, u_{2 j}\right]-\left[l_{1 j}, u_{1 j}\right]\right)=[0.09,0.11] \bigcap[0.09,0.11] \cap[0.08,0.12] \cap[0.08,0.12] \\
& =[0.09,0.11] \neq \varnothing \\
& \bigcap_{j=1}^{4}\left(\left[l_{3 j}, u_{3 j}\right]-\left[l_{1 j}, u_{1 j}\right]\right)=[0.19,0.21] \bigcap[0.18,0.21] \bigcap[0.19,0.21] \bigcap[0.18,0.21] \\
& =[0.19,0.21] \neq \varnothing \\
& \bigcap_{j=1}^{4}\left(\left[l_{4 j}, u_{4 j}\right]-\left[l_{1 j}, u_{1 j}\right]\right)=[0.29,0.31] \cap[0.28,0.32] \cap[0.28,0.32] \cap[0.29,0.31] \\
& =[0.29,0.31] \neq \varnothing
\end{aligned}
$$

Thus, $\tilde{R}$ is an additive consistency interval fuzzy preference relation. The consistency of $\tilde{R}$ can also be tested by using Eq.(15) of Theorem 4 and Eq.(28) of Theorem 6. Next we construct an additive consistent fuzzy preference relation as per the procedure in the proof of Theorem 4 .

By Eq.(21), we have

$$
p_{21}^{-}=0.09, \quad p_{21}^{+}=0.11, p_{31}^{-}=0.19, \quad p_{31}^{+}=0.21, \quad p_{41}^{-}=0.29, \quad p_{41}^{+}=0.31,
$$

let

$$
\begin{aligned}
& p_{12}^{-}=\max \{0.39,0.5-0.11,0.59-0.21,0.69-0.31\}=0.39, \\
& p_{12}^{+}=\min \{0.41,0.5-0.09,0.61-0.19,0.71-0.29\}=0.41, \\
& p_{13}^{-}=\max \{0.29,0.39-0.11,0.50-0.21,0.59-0.31\}=0.29, \\
& p_{13}^{+}=\min \{0.31,0.41-0.09,0.50-0.19,0.61-0.29\}=0.31, \\
& p_{14}^{-}=\max \{0.19,0.29-0.11,0.39-0.21,0.50-0.31\}=0.19, \\
& p_{13}^{+}=\min \{0.21,0.31-0.09,0.41-0.19,0.50-0.29\}=0.21,
\end{aligned}
$$

By Eq.(24), we have 


$$
\tilde{P}=\left[\begin{array}{cccc}
{[0.50,0.50]} & {[0.39,0.41]} & {[0.29,0.31]} & {[0.19,0.21]} \\
{[0.59,0.61]} & {[0.50,0.50]} & {[0.40,0.40]} & {[0.30,0.30]} \\
{[0.69,0.71]} & {[0.60,0.60]} & {[0.50,0.50]} & {[0.40,0.40]} \\
{[0.79,0.81]} & {[0.7,0.70]} & {[0.60,0.60]} & {[0.50,0.50]}
\end{array}\right]
$$

then by Eq.(26), we have

$$
P=\left[\begin{array}{llll}
0.5 & 0.4 & 0.3 & 0.2 \\
0.6 & 0.5 & 0.4 & 0.3 \\
0.7 & 0.6 & 0.5 & 0.4 \\
0.8 & 0.7 & 0.6 & 0.5
\end{array}\right]
$$

Obviously, $P$ is an additive consistent fuzzy preference relation, thus, $\tilde{R}$ is a consistent IFPR.

Tables 1,2 and the aforesaid process show the consistency test results using Theorems 6 and 4, respectively. It is clear that $\tilde{R}$ passes the consistency test and, hence, is a consistent IFPR. Generally speaking, Table 2 is simpler than Table 1, and consistency test calculations by Eq. (14) are the simplest. Therefore, it is recommended to employ Eq.(14) (Theorem 4) for testing consistency. As $\tilde{R}$ is additive consistent, models (M-1) and (M-2) can be applied to derive the interval weights. Alternatively, model (M-3) can also be utilized to judge whether $\tilde{R}$ is additive consistent. Setting $\beta=(n-1) / 2=1.5$ ( or $\beta=n / 2=2$ ), one can find that there exists a weight vector $W=\left(w_{1}, w_{2}, w_{3}, w_{4}\right)^{T}$ such that $J=0$, indicating that $\tilde{R}$ is a consistent IFPR. In this case, models (M-4) and (M-5) will be reduced to (M-1) and (M-2), respectively. Table 3 shows different interval weights by setting distinct values for $\beta$. It is clear that, if $\beta \rightarrow+\infty$ (for example $\beta=10000)$, then all $\tilde{w}_{i}$ 's become a constant $1 / n(0.25)$, verifying the results of Theorem 3. But in remaining cases, one has $\tilde{w}_{4} \succ \tilde{w}_{3} \succ \tilde{w}_{2} \succ \tilde{w}_{1}$. On the other hand, if $\beta<0.57$, one will have $J \neq 0$. For instance, if $\beta=0.5$, by using $\mathrm{Xu}$ and Chen's (2008b) method, one can get $J=0.07 \neq 0$, and the optimal deviation values are $d_{12}^{+}=0.0167, d_{13}^{+}=d_{14}^{+}=0.0267$, with remaining deviations being zero, implying that $\tilde{R}$ is not a consistent IFPR by Xu and Chen's additive consistency definition [59]. Wang et al. (2012) also pointed out that this IFPR does not satisfying additive or multiplicative consistency conditions as defined by $\mathrm{Xu}$ and Chen (2008b). Furthermore, if $\beta=0.57$, a unique crisp weight vector $W=(0,0.1579,0.3333,0.5088)^{T}$ is obtained by using model (M-3), which 
satisfies Eqs.(33) and, hence, (34), i.e., it satisfies Definition 3. Then, by Eq.(12), the following fuzzy preference relation can be derived

$$
R_{1}=\left[\begin{array}{cccc}
0.5 & 0.41 & 0.31 & 0.21 \\
0.59 & 0.5 & 0.4 & 0.3 \\
0.69 & 0.6 & 0.5 & 0.4 \\
0.79 & 0.7 & 0.6 & 0.5
\end{array}\right]
$$

It is evident that the fuzzy preference relation $R_{1}$ satisfies Eq.(13), i.e., IFPR $\tilde{R}$ contains an additive consistent fuzzy preference relation. If $\beta=2$, one can obtain a normalized weight vector $W=(0.1775,0.2275,0.2725,0.3225)^{T}$, and by Eq. (12), we have

$$
R_{2}=\left[\begin{array}{cccc}
0.5 & 0.4 & 0.31 & 0.21 \\
0.6 & 0.5 & 0.41 & 0.31 \\
0.69 & 0.59 & 0.5 & 0.4 \\
0.79 & 0.69 & 0.6 & 0.5
\end{array}\right]
$$

Similarly, when $\beta=2$, one can get another weight vector $W=(0.175,0.225$, $0.275,0.325)^{T}$, by Eq.(12), we have

$$
R_{3}=\left[\begin{array}{llll}
0.5 & 0.4 & 0.3 & 0.2 \\
0.6 & 0.5 & 0.4 & 0.3 \\
0.7 & 0.6 & 0.5 & 0.4 \\
0.8 & 0.7 & 0.6 & 0.5
\end{array}\right]
$$

As a matter of fact, from the interval weight vector corresponding to each $0.57<\beta<10000$ in Table 3, numerous weight vectors satisfying Eq.(33) can be obtained, then by Eq.(12), one can derive numerous corresponding fuzzy preference relations satisfying Eq.(13). This example illustrates that the additive interval consistency defined by $\mathrm{Xu}$ and Chen (2008b) needs stricter conditions than that proposed in this paper.

Example 2. $\mathrm{Xu}$ and Chen (2008b) presented a case study about three equally weighted experts $e_{k}(k=1,2,3)$ evaluating six agro-ecological regions in the province of Hubei in Central China. It is assumed that each expert furnishes his/her assessments on $x_{j}(j=1,2, \ldots, 6)$ as an IFPR $\tilde{R}^{(k)}=\left(\tilde{r}_{i j}^{(k)}\right)_{6 \times 6}(k=1,2,3)$ as follows (Xu and Chen, 2008b): 


$$
\begin{array}{r}
\tilde{R}^{(1)}=\left[\begin{array}{llllll}
{[0.5,0.5]} & {[0.5,0.7]} & {[0.7,0.8]} & {[0.5,0.6]} & {[0.6,0.7]} & {[0.8,1.0]} \\
{[0.3,0.5]} & {[0.5,0.5]} & {[0.6,0.7]} & {[0.3,0.4]} & {[0.5,0.6]} & {[0.5,0.9]} \\
{[0.2,0.3]} & {[0.3,0.4]} & {[0.5,0.5]} & {[0.3,0.5]} & {[0.4,0.5]} & {[0.6,0.8]} \\
{[0.4,0.5]} & {[0.6,0.7]} & {[0.5,0.7]} & {[0.5,0.5]} & {[0.5,0.7]} & {[0.7,0.8]} \\
{[0.3,0.4]} & {[0.4,0.5]} & {[0.5,0.6]} & {[0.3,0.5]} & {[0.5,0.5]} & {[0.4,0.8]} \\
{[0.0,0.2]} & {[0.1,0.5]} & {[0.2,0.4]} & {[0.2,0.3]} & {[0.2,0.6]} & {[0.5,0.5]}
\end{array}\right] \\
\tilde{R}^{(2)}=\left[\begin{array}{llllll}
{[0.5,0.5]} & {[0.6,0.7]} & {[0.6,0.9]} & {[0.4,0.7]} & {[0.6,0.8]} & {[0.8,0.9]} \\
{[0.3,0.4]} & {[0.5,0.5]} & {[0.5,0.8]} & {[0.4,0.5]} & {[0.5,0.7]} & {[0.5,0.9]} \\
{[0.1,0.4]} & {[0.2,0.5]} & {[0.5,0.5]} & {[0.4,0.5]} & {[0.4,0.6]} & {[0.7,0.8]} \\
{[0.3,0.6]} & {[0.5,0.6]} & {[0.5,0.6]} & {[0.5,0.5]} & {[0.6,0.7]} & {[0.7,0.9]} \\
{[0.2,0.4]} & {[0.3,0.5]} & {[0.4,0.6]} & {[0.3,0.4]} & {[0.5,0.5]} & {[0.5,0.8]} \\
{[0.1,0.2]} & {[0.1,0.5]} & {[0.2,0.3]} & {[0.1,0.3]} & {[0.2,0.5]} & {[0.5,0.5]}
\end{array}\right] \\
\tilde{R}^{(3)}=\left[\begin{array}{llllll}
{[0.5,0.5]} & {[0.4,0.6]} & {[0.5,0.7]} & {[0.4,0.7]} & {[0.6,0.8]} & {[0.7,0.8]} \\
{[0.4,0.6]} & {[0.5,0.5]} & {[0.4,0.6]} & {[0.3,0.5]} & {[0.4,0.6]} & {[0.6,0.9]} \\
{[0.3,0.5]} & {[0.4,0.6]} & {[0.5,0.5]} & {[0.4,0.6]} & {[0.4,0.7]} & {[0.5,0.8]} \\
{[0.3,0.6]} & {[0.5,0.7]} & {[0.4,0.6]} & {[0.5,0.5]} & {[0.6,0.8]} & {[0.4,0.7]} \\
{[0.2,0.4]} & {[0.4,0.6]} & {[0.3,0.6]} & {[0.2,0.4]} & {[0.5,0.5]} & {[0.6,0.7]} \\
{[0.2,0.3]} & {[0.1,0.4]} & {[0.2,0.5]} & {[0.3,0.6]} & {[0.3,0.4]} & {[0.5,0.5]}
\end{array}\right]
\end{array}
$$

$\mathrm{Xu}$ and Chen (2008b) first utilized a fuzzy weighted averaging operator $\left(\lambda=(1 / 3,1 / 3,1 / 3)^{T}\right)$ to aggregate individual IFPRs $\tilde{R}^{(k)}=\left(\tilde{r}_{i j}^{(k)}\right)_{6 \times 6} \quad(k=1,2,3)$ into a collective IFPR $\tilde{R}=\left(\tilde{r}_{i j}\right)_{6 \times 6}$ as shown below:

$$
\tilde{R}=\left[\begin{array}{llllll}
{[0.50,0.50]} & {[0.50,0.67]} & {[0.60,0.80]} & {[0.43,0.67]} & {[0.60,0.77]} & {[0.77,0.90]} \\
{[0.33,0.50]} & {[0.50,0.50]} & {[0.50,0.70]} & {[0.33,0.47]} & {[0.47,0.63]} & {[0.53,0.90]} \\
{[0.20,0.40]} & {[0.30,0.50]} & {[0.50,0.50]} & {[0.37,0.53]} & {[0.40,0.60]} & {[0.60,0.80]} \\
{[0.33,0.57]} & {[0.53,0.67]} & {[0.47,0.63]} & {[0.50,0.50]} & {[0.57,0.73]} & {[0.60,0.80]} \\
{[0.23,0.40]} & {[0.37,0.53]} & {[0.40,0.60]} & {[0.27,0.43]} & {[0.50,0.50]} & {[0.50,0.77]} \\
{[0.10,0.23]} & {[0.10,0.47]} & {[0.20,0.40]} & {[0.20,0.40]} & {[0.23,0.50]} & {[0.50,0.50]}
\end{array}\right]
$$

To test the consistency of $\tilde{R}^{(1)}, \tilde{R}^{(2)}, \tilde{R}^{(3)}$ and $\tilde{R}$, any of Theorems 4-7 can be used. To minimize the computational burden, Eq.(14) is employed. By Eq.(14), for $\tilde{R}^{(1)}$, we have:

$$
\begin{gathered}
\bigcap_{j=1}^{6}\left(\left[l_{2 j}, u_{2 j}\right]-\left[l_{1 j}, u_{1 j}\right]\right)=[-0.2,0] \cap[-0.2,0] \cap[-0.2,0] \cap[-0.3,-0.1] \cap[-0.2,0] \\
\bigcap[-0.5,0.1]=[-0.2,-0.1] \neq \varnothing
\end{gathered}
$$




$$
\begin{aligned}
& \bigcap_{j=1}^{6}\left(\left[l_{3 j}, u_{3 j}\right]-\left[l_{1 j}, u_{1 j}\right]\right)= {[-0.3,-0.2] \cap[-0.4,-0.1] \cap[-0.3,-0.2] \cap[-0.3,0] \cap } \\
& {[-0.3,-0.1] \cap[-0.4,0]=[-0.3,-0.2] \neq \varnothing } \\
& \bigcap_{j=1}^{6}\left(\left[l_{4 j}, u_{4 j}\right]-\left[l_{1 j}, u_{1 j}\right]\right)=[-0.1,0] \cap[-0.1,0.2] \cap[-0.3,0] \cap[-0.1,0] \cap[-0.2,0.1] \cap \\
& \\
& {[-0.3,0]=[-0.1,0] \neq \varnothing } \\
& \bigcap_{j=1}^{6}\left(\left[l_{5 j}, u_{5 j}\right]-\left[l_{1 j}, u_{1 j}\right]\right)=[-0.2,-0.1] \cap[-0.3,0] \cap[-0.3,-0.1] \cap[-0.3,0] \cap \\
& {[-0.2,-0.1] \cap[-0.6,0]=[-0.2,-0.1] \neq \varnothing } \\
& \bigcap_{j=1}^{6}\left(\left[l_{6 j}, u_{6 j}\right]-\left[l_{1 j}, u_{1 j}\right]\right)=[-0.5,-0.3] \cap[-0.6,0] \cap[-0.6,-0.3] \cap[-0.4,-0.2] \cap \\
& {[-0.5,0] \cap[-0.5,-0.3]=[-0.4,-0.3] \neq \varnothing }
\end{aligned}
$$

For $\tilde{R}^{(2)}$, we have:

$$
\begin{aligned}
& \bigcap_{j=1}^{6}\left(\left[l_{2 j}, u_{2 j}\right]-\left[l_{1 j}, u_{1 j}\right]\right)= {[-0.2,-0.1] \cap[-0.2,-0.1] \cap[-0.4,0.2] \cap[-0.3,0.1] \cap } \\
& {[-0.3,0.1] \cap[-0.4,0.1]=[-0.2,-0.1] \neq \varnothing } \\
& \bigcap_{j=1}^{6}\left(\left[l_{3 j}, u_{3 j}\right]-\left[l_{1 j}, u_{1 j}\right]\right)=[-0.4,-0.1] \cap[-0.5,-0.1] \cap[-0.4,-0.1] \cap[-0.3,0.1] \cap \\
& \\
& {[-0.4,0] \cap[-0.2,0]=[-0.2,-0.1] \neq \varnothing } \\
& \bigcap_{j=1}^{6}\left(\left[l_{4 j}, u_{4 j}\right]-\left[l_{1 j}, u_{1 j}\right]\right)=[-0.2,0.1] \cap[-0.2,0] \cap[-0.4,0] \cap[-0.2,0.1] \cap[-0.2,0.1] \cap \\
& {[-0.2,0.1]=[-0.2,0] \neq \varnothing } \\
& \bigcap_{j=1}^{6}\left(\left[l_{5 j}, u_{5 j}\right]-\left[l_{1 j}, u_{1 j}\right]\right)=[-0.3,-0.1] \cap[-0.4,-0.1] \cap[-0.5,0] \cap[-0.4,0] \cap \\
& {[-0.3,-0.1] \cap[-0.4,0]=[-0.3,-0.1] \neq \varnothing } \\
& \bigcap_{j=1}^{6}\left(\left[l_{6 j}, u_{6 j}\right]-\left[l_{1 j}, u_{1 j}\right]\right)=[-0.4,-0.3] \cap[-0.6,-0.1] \cap[-0.7,-0.3] \cap[-0.6,-0.1] \cap \\
& \\
& {[-0.6,-0.1] \cap[-0.4,-0.3]=[-0.4,-0.3] \neq \varnothing }
\end{aligned}
$$

For $\tilde{R}^{(3)}$, we have

$$
\begin{gathered}
\bigcap_{j=1}^{6}\left(\left[l_{2 j}, u_{2 j}\right]-\left[l_{1 j}, u_{1 j}\right]\right)=[-0.1,0.1] \cap[-0.1,0.1] \cap[-0.3,0.1] \cap[-0.4,0.1] \cap[-0.4,0] \\
\bigcap[-0.2,0.2]=[-0.1,0.1] \neq \varnothing \\
\bigcap_{j=1}^{6}\left(\left[l_{3 j}, u_{3 j}\right]-\left[l_{1 j}, u_{1 j}\right]\right)=[-0.2,0] \cap[-0.2,0.2] \cap[-0.2,0] \cap[-0.3,0.2] \cap[-0.4,0.1] \\
\bigcap[-0.3,0.1]=[-0.2,0] \neq \varnothing
\end{gathered}
$$




$$
\begin{gathered}
\bigcap_{j=1}^{6}\left(\left[l_{4 j}, u_{4 j}\right]-\left[l_{1 j}, u_{1 j}\right]\right)=[-0.2,0.1] \cap[-0.1,0.3] \cap[-0.3,0.1] \cap[-0.2,0.1] \cap \\
{[-0.2,0.2] \cap[-0.4,0]=[-0.2,0] \neq \varnothing} \\
\bigcap_{j=1}^{6}\left(\left[l_{5 j}, u_{5 j}\right]-\left[l_{1 j}, u_{1 j}\right]\right)=[-0.3,-0.1] \cap[-0.2,0.2] \cap[-0.4,0.1] \cap[-0.5,0] \cap \\
{[-0.3,-0.1] \cap[-0.2,0]=[-0.2,0] \neq \varnothing} \\
\bigcap_{j=1}^{6}\left(\left[l_{6 j}, u_{6 j}\right]-\left[l_{1 j}, u_{1 j}\right]\right)=[-0.3,-0.2] \cap[-0.5,0] \cap[-0.5,0] \cap[-0.4,0.2] \cap \\
{[-0.5,-0.2] \cap[-0.3,-0.2]=[-0.3,-0.2] \neq \varnothing}
\end{gathered}
$$

For $\tilde{R}$, we have:

$$
\begin{aligned}
& \bigcap_{j=1}^{6}\left(\left[l_{2 j}, u_{2 j}\right]-\left[l_{1 j}, u_{1 j}\right]\right)= {[-0.17,0] \cap[-0.17,0] \cap[-0.3,0.1] \cap[-0.34,0.04] \cap } \\
& {[-0.3,0.03] \cap[-0.37,0.13]=[-0.17,0] \neq \varnothing } \\
& \bigcap_{j=1}^{6}\left(\left[l_{3 j}, u_{3 j}\right]-\left[l_{1 j}, u_{1 j}\right]\right)=[-0.3,-0.1] \cap[-0.23,0] \cap[-0.3,-0.1] \cap[-0.4,0.1] \cap \\
& \\
& {[-0.23,0] \cap[-0.3,0.03]=[-0.23,-0.1] \neq \varnothing } \\
& \bigcap_{j=1}^{6}\left(\left[l_{4 j}, u_{4 j}\right]-\left[l_{1 j}, u_{1 j}\right]\right)=[-0.17,0.07] \cap[-0.14,0.17] \cap[-0.33,0.03] \cap \\
& {[-0.17,0.07] \cap[-0.2,0.13] \cap[-0.3,0.03]=[-0.14,0.03] \neq \varnothing } \\
& \bigcap_{j=1}^{6}\left(\left[l_{5 j}, u_{5 j}\right]-\left[l_{1 j}, u_{1 j}\right]\right)=[-0.27,-0.1] \cap[-0.3,0.03] \cap[-0.4,0] \cap[-0.4,0] \cap \\
& {[-0.27,-0.1] \cap[-0.4,0]=[-0.27,-0.1] } \\
& \bigcap_{j=1}^{6}\left(\left[l_{6 j}, u_{6 j}\right]-\left[l_{1 j}, u_{1 j}\right]\right)= {[-0.4,-0.27] \cap[-0.57,-0.03] \cap[-0.6,-0.2] \cap } \\
& {[-0.47,-0.03] \cap[-0.54,-0.1] \cap[-0.4,-0.23]=[-0.4,-0.27] \neq \varnothing }
\end{aligned}
$$

The aforesaid computational analysis indicates that $\tilde{R}^{(1)}, \tilde{R}^{(2)}, \tilde{R}^{(3)}$ and $\tilde{R}$ are all additive consistent IFPRs. As such, (M-1) and (M-2) can be employed to derive interval weights $\tilde{w}_{i}(i=1,2, \ldots, 6)$. To facilitate a comparative study with the approach in $\mathrm{Xu}$ and Chen (2008b), we only compute the interval weights for the collective IFPR $\tilde{R}$. The determination of interval weights for $\tilde{R}^{(1)}, \tilde{R}^{(2)}$, $\tilde{R}^{(3)}$ can be carried out in the same way and, hence, is omitted here. Table 4 displays the interval weights of $\tilde{R}$ by setting different values for $\beta$. If model (M-3) is entertained, one can confirm that $J=0$ and optimal deviation variables are equal to zero when $\beta \geq 0.6$, certifying that $\tilde{R}$ is a consistent IFPR. Table 4 
shows that the lower bound $w_{i}^{-}$and the upper bound $w_{i}^{+}$of $\tilde{w}_{i}$ are equal when $\beta=0.6$, When the value of $\beta$ increases, the dispersion between $w_{i}^{-}$and $w_{i}^{+}$first increases, then gradually decreases. When $\beta \rightarrow+\infty$, the dispersion decreases to zero, and all the $\tilde{w}_{i}(i=1,2, \ldots, 6)$ tend to be equal ( i.e., $\left.\tilde{w}_{i}=1 / 6=0.1667\right)$. By Eq.(36), we have $\tilde{w}_{1} \succ \tilde{w}_{4} \succ \tilde{w}_{2} \succ \tilde{w}_{3} \succ \tilde{w}_{5} \succ \tilde{w}_{6}$ in all the cases. Furthermore, if $\beta=0.6$, applying model (M-3) yields a crisp weight vector $W=(0.45,0.1667,0.1667,0.2167,0,0)^{T}$, which satisfies Eqs.(33) (and, hence, (34)), i.e., it satisfies Definition 3. Then, by Eq.(12), the following fuzzy preference relation can be obtained:

Table 4. Interval weights of $\tilde{R}$

\begin{tabular}{lllllll}
\hline$\beta$ & $\tilde{w}_{1}$ & $\tilde{w}_{2}$ & $\tilde{w}_{3}$ & $\tilde{w}_{4}$ & $\tilde{w}_{5}$ & $\tilde{w}_{6}$ \\
\hline 0.6 & {$[0.45,0.45]$} & {$[0.1667,0.1667]$} & {$[0.1667,0.1667]$} & {$[0.2167,0.2167]$} & {$[0,0]$} & {$[0,0]$} \\
3 & {$[0.1911,0.2361]$} & {$[0.1539,0.1928]$} & {$[0.1411,0.18]$} & {$[0.1733,0.2122]$} & {$[0.1261,0.175]$} & {$[0.085,0.1333]$} \\
10 & {$[0.174,0.1875]$} & {$[0.1628,0.1745]$} & {$[0.159,0.1707]$} & {$[0.1687,0.1803]$} & {$[0.1545,0.1692]$} & {$[0.1422,0.1567]$} \\
100 & {$[0.1674,0.1688]$} & {$[0.1663,0.1675]$} & {$[0.1659,0.1671]$} & {$[0.1669,0.1680]$} & {$[0.1655,0.1669]$} & {$[0.1642,0.1657]$} \\
1000 & {$[0.1667,0.1669]$} & {$[0.1666,0.1667]$} & {$[0.1666,0.1667]$} & {$[0.1667,0.1668]$} & {$[0.1665,0.1667]$} & {$[0.1664,0.1666]$} \\
\hline
\end{tabular}

$$
R_{1}=\left[\begin{array}{cccccc}
0.5 & 0.67 & 0.67 & 0.64 & 0.77 & 0.77 \\
0.33 & 0.5 & 0.5 & 0.47 & 0.6 & 0.6 \\
0.33 & 0.5 & 0.5 & 0.47 & 0.6 & 0.6 \\
0.36 & 0.53 & 0.53 & 0.5 & 0.63 & 0.63 \\
0.23 & 0.4 & 0.4 & 0.37 & 0.5 & 0.5 \\
0.23 & 0.4 & 0.4 & 0.37 & 0.5 & 0.5
\end{array}\right]
$$

It is apparent that the fuzzy preference relation $R_{1}$ satisfies Eq.(13), or the IFPR $\tilde{R}$ contains an additive consistent fuzzy preference relation. If $\beta=3$, one can get a normalized weight vector $W=(0.2,0.19,0.1633,0.2,0.1467,0.1)^{T}$, and by Eq.(12), we have

$$
R_{2}=\left[\begin{array}{cccccc}
0.5 & 0.53 & 0.6101 & 0.5 & 0.6599 & 0.8 \\
0.47 & 0.5 & 0.5801 & 0.47 & 0.6299 & 0.77 \\
0.3899 & 0.4199 & 0.5 & 0.3899 & 0.5498 & 0.6899 \\
0.5 & 0.53 & 0.6101 & 0.5 & 0.6599 & 0.8 \\
0.3401 & 0.3701 & 0.4502 & 0.3401 & 0.5 & 0.6401 \\
0.2 & 0.23 & 0.3101 & 0.2 & 0.3599 & 0.5
\end{array}\right]
$$

Similarly, numerous weight vectors satisfying Eq.(33) can be obtained, then by Eq.(12), one can get numerous corresponding fuzzy preference relations satisfying 
Eq.(13). This confirms that the collective IFPR $\tilde{R}$ is consistent. Given that $\tilde{R}$ is a weighted average of additive consistent IFPRs $\tilde{R}_{1}, \tilde{R}_{2}, \tilde{R}_{3}$, Theorem 10 is also verified. However, if $\beta<0.6$, then one will get $J \neq 0$. For example, if $\beta=0.5$, Xu and Chen (2008b) got $J=0.05$ by solving the model (M-3), and the optimal deviation values are $d_{16}^{+}=0.025, d_{36}^{-}=0.025$, and other deviations are equal to zero. Based on the consistency definition in $\mathrm{Xu}$ and Chen $(2008 \mathrm{~b}), \tilde{R}$ is not an additive consistent IFPR. This example illustrates that $\mathrm{Xu}$ and Chen's definition of additive consistency is more restrictive than the one presented in this paper.

Remark 7. In the above computation, interval weights are only determined for the collective IFPR $\tilde{R}$ in order to compare with $\mathrm{Xu}$ and Chen's results in (Xu and Chen, 2008b). Similarly, one can compute individual interval weights for $\tilde{R}_{1}$, $\tilde{R}_{2}$ and $\tilde{R}_{3}$ by setting $\beta \geq 1$ for $\tilde{R}_{1}, \beta \geq 1$ for $\tilde{R}_{2}, \beta \geq 0.9$ for $\tilde{R}_{3}$, respectively.

\section{Conclusions}

Motivated by the research in $\mathrm{Xu}$ and Chen (2008b), this paper investigates how to derive interval weights from an IFPR. New properties are derived for additive consistent fuzzy preference relations and a correspondence relationship is established between the element $r_{i j}$ of an additive fuzzy preference relation and a weight vector $W=\left(w_{1}, w_{2}, \ldots, w_{n}\right)^{T}$. Some simple yet practical approaches are developed to check whether an IFPR is additive consistent. Based on the correspondence between the element $r_{i j}$ and the weight vector $W=\left(w_{1}, w_{2}\right.$, $\left.\ldots, w_{n}\right)^{T}$, additive consistency is extended to IFPRs, and a revised definition is put forward. Optimization models are then established to generate interval priority weights from additive consistent or inconsistent IFPRs. A practical procedure is presented to solve group decision problems with IFPRs assessment data. To illustrate the proposed methods, two numerical examples are adapted from existing literature and a comparative study is conducted between the proposed methods and those given in $\mathrm{Xu}$ and Chen (2008b). Numerical experiment by appropriately adjusting the value of a parameter $\beta$ indicates that one can find an 
additive consistent fuzzy preference relation contained in a given IFPR, but the method in Xu and Chen (2008b) fails to identify such a fuzzy preference relation. Furthermore, if an IFPR is additive consistent, different weight vectors can be obtained by setting different $\beta$ without affecting the ranking order.

The proposed approaches assume that IFPRs provided by DMs are complete. However, in real decision situations, there may be cases where DMs express their judgments by using preference relations with incomplete information (Alonso et al., 2004; 2008; Fedrizzi and Silvio, 2007; Genç et al., 2010; Herrera-Viedma et al., 2007a; 2007b; Lee et al., 2007; Liu et al., 2012b; Xu et al., 2010; Xu, 2004b; 2004a; Xu and Chen, 2008a; Xu and Da, 2008; 2009; Chiclana et al., 2008; Gong, 2008; Alonso et al., 2009; Alonso et al., 2010; Cabrerizo et al., 2010a; Chiclana et al., 2009a; Xu et al., 2013a; Xu and Wang, 2013). It is a worthy topic to examine how the proposed models can be modified to accommodate incomplete IFPRs.

\section{Acknowledgment}

The authors are very grateful to the Associate Editor and the two anonymous reviewers for their constructive comments and suggestions that have further helped to improve the quality and presentation of this paper. Yejun $\mathrm{Xu}$ would like to acknowledge the financial support of a Grant (No. 71101043) from National Natural Science of China. Kevin W. Li would like to acknowledge the financial support of a Natural Sciences and Engineering Research Council of Canada (NSERC) Discovery Grant, and Grants (No.71272129 and 71271188) from National Natural Science Foundation of China. Huimin Wang would like to acknowledge the financial support of a Grant (No.12\&ZD214) from Major Program of the National Social Science Foundation of China.

\section{References}

Alonso, S., Cabrerizo, F. J., Chiclana, F., Herrera, F. \& Herrera-Viedma, E. (2009) Group decision making with incomplete fuzzy linguistic preference relations. Int J Intell Syst 24(2): 201222.

Alonso, S., Chiclana, F., Herrera, F. \& Herrera-Viedma, E. (2004) A learning procedure to estimate missing values in fuzzy preference relations based on additive consistency. In MDAI2004,LNAI Vol. 3131, 227-238 (Eds V. Torra and Y. Narukawa). Berlin Heidelberg: Springer-Verlag 
Alonso, S., Chiclana, F., Herrera, F., Herrera-Viedma, E., Alcalá-Fdez, J. \& Porcel, C. (2008) A consistency-based procedure to estimate missing pairwise preference values. Int $\mathrm{J}$ Intell Syst 23: 155-175.

Alonso, S., Herrera-Viedma, E., Chiclana, F. \& Herrera, F. (2010) A web based consensus support system for group decision making problems and incomplete preferences. Inform Sci 180: 4477-4495.

Cabrerizo, F. J., Heradio, R., Pérez, I. J. \& Herrera-Viedma, E. (2010a) A selection process based on additive consistency to deal with incomplete fuzzy linguistic information. J Univers Comput Sci 16(1): 62-81.

Cabrerizo, F. J., Perez, I. J. \& Herrera-Viedma, E. (2010b) Managing the consensus in group decision making in an unbalanced fuzzy linguistic context with incomplete information. Knowl-Based Syst 23(2): 169-181.

Chiclana, F., Herrera-Viedma, E. \& Alonso, S. (2009a) A note on two methods for estimating missing pairwise preference values. IEEE Trans Syst Man Cybern B: Cybern 39(6): 1628-1633.

Chiclana, F., Herrera-Viedma, E., Alonso, S. \& Herrera, F. (2008) A note on the estimation of missing pairwise preference values: A U-consistency based method. Int J Uncertainty, Fuzzy Knowledge-Based Syst 16(2): 19-32.

Chiclana, F., Herrera-Viedma, E., Alonso, S. \& Herrera, F. (2009b) Cardinal consistency of reciprocal preference relations: A characterization of multiplicative transitivity. IEEE Trans Fuzzy Syst 17(1): 14-23.

Chiclana, F., Herrera-Viedma, E., Herrera, F. \& Alonso, S. (2007) Some induced ordered weighted averaging operators and their use for solving group decision-making problems based on fuzzy preference relations. Eur J Oper Res 182: 383-399.

Chiclana, F., Herrera, E. \& Herrera-Viedma, E. (2001) Integrating multiplicative preference relations in a multipurpose decision-making based on fuzzy preference relations. Fuzzy Sets Syst 122: 277-291.

Chiclana, F., Herrera, F. \& Herrera-Viedma, E. (1998) Integrating three representation models in fuzzy multipurpose decision making based on fuzzy preference relations. Fuzzy Sets Syst 97: 33-48.

Chiclana, F., Herrera, F. \& Herrera-Viedma, E. (2002) A note on the internal consistency of various preference representations. Fuzzy Sets Syst 131: 75-78.

Chiclana, F., Herrera, F., Herrera-Viedma, E. \& Martínez, L. (2003) A note on the reciprocity in the aggregation of fuzzy preference relations using OWA operators. Fuzzy Sets Syst 137: 71-83.

Dong, Y. C., Xu, Y. F. \& Li, H. Y. (2008) On consistency measures of linguistic preference relations. Eur J Oper Res 189: 430-444.

Fan, Z. P., Ma, J., Jiang, Y. P., Sun, Y. H. \& Ma, L. (2006) A goal programming approach to group decision making based on multiplicative preference relations and fuzzy preference relations. Eur J Oper Res 174: 311-321.

Fedrizzi, M. \& Brunelli, M. (2009) On the normalisation of a priority vector associated with a reciprocal relation. Int J Gen Syst 38(5): 579-586.

Fedrizzi, M. \& Brunelli, M. (2010) On the priority vector associated with a reciprocal relation and a pairwise comparison matrix. Soft Comput 14: 639-645.

Fedrizzi, M. \& Silvio, G. (2007) Incomplete pairwise comparison and consistency optimization. Eur J Oper Res 183: 303-313.

Genç, S., Boran, F. E., Akay, D. \& Xu, Z. S. (2010) Interval multiplicative transitivity for consistency, missing values and priority weights of interval fuzzy preference relations. Inform Sci 180: 4877-4891.

Gong, Z. W. (2008) Least-square method to priority of the fuzzy preference relations with incomplete information. Int J Approx Reason 47: 258-264.

Herrera-Viedma, E., Alonso, S., Chiclana, F. \& Herrera, F. (2007a) A consensus model for group decision making with incomplete fuzzy preference relations. IEEE Trans Fuzzy Syst 15(5): 863-877.

Herrera-Viedma, E., Chiclana, F., Herrera, F. \& Alonso, S. (2007b) Group decision-making model with incomplete fuzzy preference relations based on additive consistency. IEEE Trans Syst Man Cybern B: Cybern 37(1): 176-189.

Herrera-Viedma, E., Herrera, F., Chiclana, F. \& Luque, M. (2004) Some issues on consistency of fuzzy preference relations. Eur J Oper Res 154: 98-109.

Herrera, F., Martíze, L. \& Sánchez, P. J. (2005) Managing non-homogeneous information in group decision making. Eur J Oper Res 166: 115-132. 
Jiang, Y. L. (2007) An approach to group decision making based on interval fuzzy relations. J Syst Science Syst Eng 16: 113-120.

Kacprzyk, J. (1986) Group decision making with a fuzzy linguistic majority. Fuzzy Sets Syst 18: $105-118$.

Lan, J. B., Hu, M. M., Ye, X. M. \& Sun, S. Q. (2012) Deriving interval weights from an interval multiplicative consistent fuzzy preference relation. Knowl-Based Syst 26: 128-134.

Lee, H. S., Chou, M. T., Fang, H. H., Tseng, W. K. \& Yeh, C. H. (2007) Estimating missing values in incomplete additive fuzzy preference relations. In KES 2007/ WIRN 2007, LNAI, Vol. 4693, 1307-1314 (Ed B. Apolloni).

Lee, H. S. \& Tseng, W. K. (2006) Goal programming methods for constructing additive consistency fuzzy preference relations. In KES 2006, Part II, LNAI 4252, 910-916 (Eds B. Gabrys, R. J. Howlett and L. C. Jain). Springer-Verlag Berlin Heidelberg.

Liu, F., Zhang, W. G. \& Fu, J. H. (2012a) A new method of obtaining the priority weights from an interval fuzzy preference relation. Inform Sci 185: 32-42.

Liu, X. W., Pan, Y. W., Xu, Y. J. \& Yu, S. (2012b) Least square completion and inconsistency repair methods for additively consistent fuzzy preference relations. Fuzzy Sets Syst 198: $1-19$

Orlovsky, S. A. (1978) Decision-making with a fuzzy preference relations. Fuzzy Sets Syst 1: 155167.

Shen, P. D., Chyr, W. L., Lee, H. S. \& Lin, K. (2009)Correspondence between incomplete fuzzy preference relation and its priority vector. In KES 2009 Part II, LNAI 5712, 745-751 (Ed J. D. Velásquez). Berlin Heidelberg: Springer-Verlag

Tanino, T. (1984) Fuzzy preference orderings in group decision making. Fuzzy Sets Syst 12: 117131.

Wang, J., Lan, J. B., Ren, P. Y. \& Luo, Y. Y. (2012) Some programming models to derive priority weights from additive interval fuzzy preference relation. Knowl-Based Syst 27: 69-77.

Wang, T. C. \& Chen, Y. H. (2008) Applying fuzzy linguistic preference relations to the improvement of consistency of fuzzy AHP. Inform Sci 178: 3755-3765.

Wang, Y. M., Yang, J. B. \& Xu, D. L. (2005a) Interval weight generation approaches based on consistency test and interval comparison matrices. Appl Math Comput 167: 252-273.

Wang, Y. M., Yang, J. B. \& Xu, D. L. (2005b) A two-stage logarithmic goal programming method for generating weights from interval comparison matrices. Fuzzy Sets Syst 152: 475-498.

Wang, Z. J. \& Li, K. W. (2012) Goal programming approaches to deriving interval weights based on interval fuzzy preference relations. Inform Sci 193: 180-198.

Wu, Z. B. \& Xu, J. P. (2012) A concise consensus support model for group decision making with reciprocal preference relations based on deviation measures. Fuzzy Sets Syst 206: 58-73.

Xu, Y. J. \& Da, Q. L. (2008) Weighted least-square method and its improvment for priority of incomplete complementary judgement matrix. Syst Eng Electro 30(7): 1273-1276.

Xu, Y. J. \& Da, Q. L. (2009) Methods for priority of incomplete complementary judgement matrices. Syst Eng Electro 31(1): 95-99.

Xu, Y. J., Da, Q. L. \& Liu, L. H. (2009) Normalizing rank aggregation method for priority of a fuzzy preference relation and its effectiveness. Int J Approx Reason 50: 1287-1297.

Xu, Y. J., Da, Q. L. \& Wang, H. M. (2010) A note on group decision-making procedure based on incomplete reciprocal relations. Soft Comput 15(7): 1289-1300.

Xu, Y. J., Patnayakuni, R. \& Wang, H. M. (2013a) Logarithmic least squares method to priority for group decision making with incomplete fuzzy preference relations. Appl Math Model 37(4): 2139-2152.

Xu, Y. J., Patnayakuni, R. \& Wang, H. M. (2013b) The ordinal consistency of a fuzzy preference relation. Inform Sci 224(1): 152-164.

Xu, Y. J. \& Wang, H. M. (2013) Eigenvector method, consistency test and inconsistency repairing for an incomplete fuzzy preference relation. Appl Math Model 37(7): 5171-5183.

Xu, Z. S. (2000) Generazlied fuzzy consistent matrix and its priority method. J PLA Univ Sci Tech 1(6): 97-99.

Xu, Z. S. (2004a) Goal programming models for obtaining the priority vector of incomplete fuzzy preference relation. Int J Approx Reason 36: 261-270.

Xu, Z. S. (2004b) Incomplete complementary judgement matrix. Syst Eng-Theory \& Practice 25: 93-97.

Xu, Z. S. (2004c) On compatibility of interval fuzzy preference relations. Fuzzy Optim Decis Making 3: 217-225.

Xu, Z. S. \& Chen, J. (2008a) Group decision-making procedure based on incomplete reciprocal relations. Soft Comput 12: 515-521. 
Xu, Z. S. \& Chen, J. (2008b) Some models for deriving the priority weights from interval fuzzy preference relations. Eur J Oper Res 184: 266-280.

Yao, M. \& Zhang, S. (1997) Fuzzy consistent matrix and its applications in soft science. System Engineering 15(2): 54-57. 